\title{
MID-INFRARED COLORS OF DWARF GALAXIES: YOUNG STARBURSTS MIMICKING ACTIVE GALACTIC NUCLEI
}

\author{
Kevin N. Hainline ${ }^{1}$, Amy E. Reines ${ }^{2,5}$, Jenny E. Greene ${ }^{3}$, and Daniel Stern ${ }^{4}$ \\ ${ }^{1}$ Steward Observatory, University of Arizona, 933 North Cherry Avenue, Tucson, AZ 85721, USA \\ ${ }^{2}$ National Optical Astronomy Observatory, Tucson, AZ 85726, USA \\ ${ }^{3}$ Department Astrophysical Sciences, Princeton University, Princeton, NJ 08544, USA \\ ${ }^{4}$ Jet Propulsion Laboratory, California Institute of Technology, 4800 Oak Grove Drive, Mail Stop 169-221, Pasadena, CA 91109, USA \\ Received 2016 June 23; revised 2016 September 2; accepted 2016 September 20; published 2016 November 22
}

\begin{abstract}
Searching for active galactic nuclei (AGNs) in dwarf galaxies is important for our understanding of the seed black holes that formed in the early universe. Here, we test infrared selection methods for AGN activity at low galaxy masses. Our parent sample consists of $\sim 18,000$ nearby dwarf galaxies $\left(M_{*}<3 \times 10^{9} M_{\odot}, z<0.055\right)$ in the Sloan Digital Sky Survey with significant detections in the first three bands of the AllWISE data release from the Widefield Infrared Survey Explorer (WISE). First, we demonstrate that the majority of optically selected AGNs in dwarf galaxies are not selected as AGNs using WISE infrared color diagnostics and that the infrared emission is dominated by the host galaxies. We then investigate the infrared properties of optically selected star-forming dwarf galaxies, finding that the galaxies with the reddest infrared colors are the most compact, with blue optical colors, young stellar ages, and large specific star formation rates. These results indicate that great care must be taken when selecting AGNs in dwarf galaxies using infrared colors, as star-forming dwarf galaxies are capable of heating dust in such a way that mimics the infrared colors of more luminous AGNs. In particular, a simple $W 1-W 2$ color cut alone should not be used to select AGNs in dwarf galaxies. With these complications in mind, we present a sample of $41 \mathrm{dwarf}$ galaxies that fall in the WISE infrared color space typically occupied by more luminous AGNs and that are worthy of follow-up observations.
\end{abstract}

Key words: cosmology: observations - galaxies: dwarf - galaxies: evolution - galaxies: nuclei

\section{INTRODUCTION}

There is now an overwhelming body of evidence suggesting that all massive galaxies host a central supermassive black hole that grows alongside the stellar population (Kormendy \& Richstone 1995; Kormendy \& Ho 2013). In addition, a relationship has been observed between the central black hole mass and the galaxy bulge stellar velocity dispersion that spans many orders of magnitude (Ferrarese \& Merritt 2000; Gebhardt et al. 2000). The origin of this relationship is still not well understood, but current theories imply that galaxy mergers and interactions play a role, both in increasing the stellar mass of a galaxy and in driving gas toward the centers of galaxies, feeding black holes (Hopkins et al. 2008; Koss et al. 2010; Ellison et al. 2011; Bessiere et al. 2012; Sabater et al. 2013). However, this view of galaxy growth implies the existence of low-mass "seed" black holes that must have existed at high redshift. To understand these difficult-to-observe objects, researchers have turned to observations of nearby dwarf galaxies that may host analogous lower-mass black holes (for a review, see Reines \& Comastri 2016). By assembling large samples of low-mass black holes, it may be possible to distinguish between the different proposed theoretical scenarios for their creation: these objects may be remnants of massive Population III stars (Bromm \& Yoshida 2011), a result of direct collapse of primordial dense gas (Haehnelt \& Rees 1993; Begelman et al. 2006; Lodato \& Natarajan 2006; van Wassenhove et al. 2010), or perhaps they are the end product of very massive stars formed through stellar mergers in dense star clusters (Gürkan et al. 2004; Freitag et al. 2006; Goswami et al. 2012; Giersz et al. 2015; Lützgendorf et al. 2016).

\footnotetext{
${ }^{5}$ Hubble Fellow.
}

Assembling these large samples of low-mass black holes is made difficult by the fact that resolving their gravitational sphere of influence is currently not feasible at distances larger than a few megaparsecs. However, active galactic nucleus (AGN) emission across the electromagnetic spectrum can be used to infer the existence of a black hole. Observations at optical wavelengths have been used to uncover AGNs in NGC 4395 (Filippenko \& Sargent 1989; Filippenko \& Ho 2003) and POX 52 (Barth et al. 2004), while data at X-ray and radio wavelengths have been used to find AGNs in both Henize 2-10 (Reines et al. 2011; Reines \& Deller 2012) and the dwarf galaxy pair Mrk 709 (Reines et al. 2014). Larger samples of low-mass AGNs have been uncovered at optical (Greene \& Ho 2004, 2007; Dong et al. 2012; Reines et al. 2013; Moran et al. 2014) and X-ray (Lemons et al. 2015; Mezcua et al. 2015; Pardo et al. 2016) wavelengths, which have been targeted with successful follow-up observations (Baldassare et al. 2016), including the discovery of a $5 \times 10^{4} M_{\odot}$ BH in RGG 118 (Baldassare et al. 2015).

One important and often-used method for selecting luminous AGNs relies on observations made in the mid-IR, where dust, heated by the central accreting black hole, reprocesses the light and emits with a characteristic red IR power-law spectrum. Infrared emission only minimally suffers from nuclear and galaxyscale obscuration, and so mid-IR observations have successfully uncovered large numbers of unobscured and obscured luminous AGNs and quasars (Lacy et al. 2004, 2013; Stern et al. 2005, 2012; Hickox et al. 2007; Donley et al. 2008; Ashby et al. 2009; Assef et al. 2010; Mendez et al. 2013; Hainline et al. 2014). The all-sky mid-IR coverage of the Wide-field Infrared Survey Explorer (WISE, Wright et al. 2010) has allowed for observations of large samples of objects, and multiple authors have proposed 
WISE color schemes that select for the red AGN power-law emission in the infrared (Jarrett et al. 2011; Mateos et al. 2012; Stern et al. 2012). These selection methods rely on the fact that AGNs are capable of heating dust to temperatures well above what is observed from stellar processes in moderate to high-mass galaxies, and have demonstrated high levels of reliability when applied to these objects.

Recently, Satyapal et al. (2014) and Sartori et al. (2015) used mid-IR selection methods to assemble large samples of low-mass galaxy AGN candidates from WISE data. Under the assumption that WISE selection targets optically obscured, "hidden" AGNs in these objects, these authors draw broad conclusions about the population of low-mass black holes. The Satyapal et al. (2014) study targets "bulgeless" galaxies without optical evidence for an AGN, and the authors conclude that star formation is not the primary source of the IR emission in their sample. In addition, they propose that the fraction of galaxies hosting IR-selected AGN activity increases at low masses. This puzzling trend was also seen (with lower significance), using a larger sample of dwarf galaxies, by Sartori et al. (2015), who compared multiple AGN selection methods and concluded that dwarf galaxies with WISE colors indicative of AGN activity are bluer and potentially may have more ongoing star formation and lower metallicities than those selected using optical emission lines.

These results are intriguing in light of the fact that no other tracer of AGN activity has thus far uncovered such large samples of AGNs in dwarf galaxies. In most AGN selection regimes, more luminous AGNs are easier to find both because massive BHs have a higher Eddington limit and because of increasing confusion due to star formation at low mass. Thus, the observed increase in AGN fraction at the lowest dwarf galaxy masses is puzzling. Low-mass SMBHs that exist in dwarf galaxies power AGNs that have such low luminosities that star formation in their hosts becomes a significant source of contamination. In particular, it has been shown that lowmetallicity dwarf starburst galaxies are capable of heating dust to very high temperatures (Hirashita \& Hunt 2004; Reines et al. 2008; Griffith et al. 2011; Izotov et al. 2011, 2014; RémyRuyer et al. 2015), producing red mid-IR colors. This was recently explored in O'Connor et al. (2016), who found that galaxies with low stellar masses have predominantly red WISE colors, which the authors associate with higher specific star formation rates (sSFRs) in these galaxies. Thus, it may be that using common mid-IR AGN selection methods on dwarf galaxies results in the selection of a large number of starforming galaxies that contaminate the samples, leading to erroneous conclusions about AGN fractions at these masses.

In this paper, we use WISE data to empirically examine the infrared colors of dwarf galaxies as a function of their properties as probed by Sloan Digital Sky Survey (SDSS) data. Our results suggest that star formation, and not AGN activity, is responsible for the red WISE colors for the majority of the dwarf galaxy population (also see Izotov et al. 2014). We propose a small sample of dwarf galaxy AGN candidates that require follow-up observations to confirm black hole activity.

In Section 2, we discuss our sample selection and describe how these objects were matched to WISE photometry. We start by exploring the infrared properties of dwarf galaxies that have optical spectroscopic evidence for an AGN in Section 3.1. We then investigate optically selected star-forming galaxies and propose a sample of IR-selected AGN candidates in Section 3.2. We expand our sample to include IR-selected AGN candidates without optical emission-line flux measurements in Section 3.3, after which we compare these objects to samples of IR-selected AGN candidates presented in the literature in Section 3.4. Finally, we explore our WISE detection limits in Section 3.5, and we discuss our results and draw conclusions in Section 4.

\section{SAMPLE SELECTION AND DATA}

The dwarf galaxies we explore in this paper were selected from the NASA Sloan Atlas (NSA), a catalog of galaxies at $z<0.055$ selected from the SDSS DR8 (York \& SDSS Collaboration 2000; Aihara et al. 2011), where the observations were re-analyzed, resulting in improved photometry (Blanton et al. 2011) and spectroscopy (Yan 2011; Yan \& Blanton 2012). In addition, stellar masses for each object were derived with the kcorrect code of Blanton \& Roweis (2007), which uses the stellar population synthesis models of Bruzual \& Charlot (2003) and the nebular emission-line models of Kewley et al. (2001). These masses are provided in the NSA in units of $M_{\odot} h^{-2}$, and in this paper we have assumed $h=0.73$.

Following Reines et al. (2013), we selected all galaxies with $M_{*}<3 \times 10^{9} M_{\odot}$, the approximate mass of the Large Magellanic Cloud (van der Marel et al. 2002). We matched those objects to the AllWISE data release, which provides increased sensitivity compared to the previous WISE All-sky data release. ${ }^{6}$ We note that because of the polar orbit of the WISE telescope, the total exposure time across the full sky is non-uniform and depends on both the sky position and the zodiacal foreground emission. As the $W 1-W 2$ color forms the basis for both of the primary AGN selection criteria that we will explore in this study (that of Jarrett et al. 2011 and Stern et al. 2012), and given the importance of deep data at $\lambda<5 \mu \mathrm{m}$ for separating emission from dust heated by star formation and AGN activity, we chose to use the updated AllWISE data, with $W 1(3.4 \mu \mathrm{m})$ and $W 2(4.6 \mu \mathrm{m}) 5 \sigma$ sensitivities of $54 \mu \mathrm{Jy}$ and $71 \mu \mathrm{Jy}$, respectively (compared to $68 \mu \mathrm{Jy}$ and $111 \mu \mathrm{Jy}$ in the All-sky data release). These sensitivities are the minimum depth of the survey estimated for low coverage sky away from the Galactic plane.

When cross-matching with the AllWISE catalog, we used a 5 " matching radius, and then chose the specific WISE photometry for each object based on the WISE ext_flg value. Following the AllWISE Explanatory Supplement, ${ }^{\overline{6}}$ for most objects (ext_flg $=0$ or 4 ), we chose the "profile-fitting" photometric magnitudes, which are optimized for objects that are unresolved in WISE. For those objects where ext_flg $=1,2$, or 3, we used the "standard" aperture magnitudes, which better traces the photometry for objects that are resolved. Finally, for a small subset of objects with 2MASS photometry, where ext_flg $=5$, we used the photometry using apertures scaled from the 2MASS XSC shape values. Each object was only included in our sample if the signal-to-noise ratio $(\mathrm{S} / \mathrm{N})$ for the W1-, W2-, and W3-band photometry was greater than 3, yielding a sample of 18,482 dwarf galaxies with significant WISE detections. The majority of the objects were removed from our final sample because of their low $\mathrm{S} / \mathrm{N} \mathrm{W3}$ photometry ( $~ 58 \%$ of the total NSA sample, compared to $\sim 4 \%$ and $\sim 7 \%$ for $W 1$ and $W 2$, respectively). The median $W 1-W 2$ and $W 2-W 3$ color uncertainties are 0.07 and 0.19 , respectively, which we show with error bars in our WISE color-color diagrams.

\footnotetext{
http://wise2.ipac.caltech.edu/docs/release/allwise/expsup/
} 


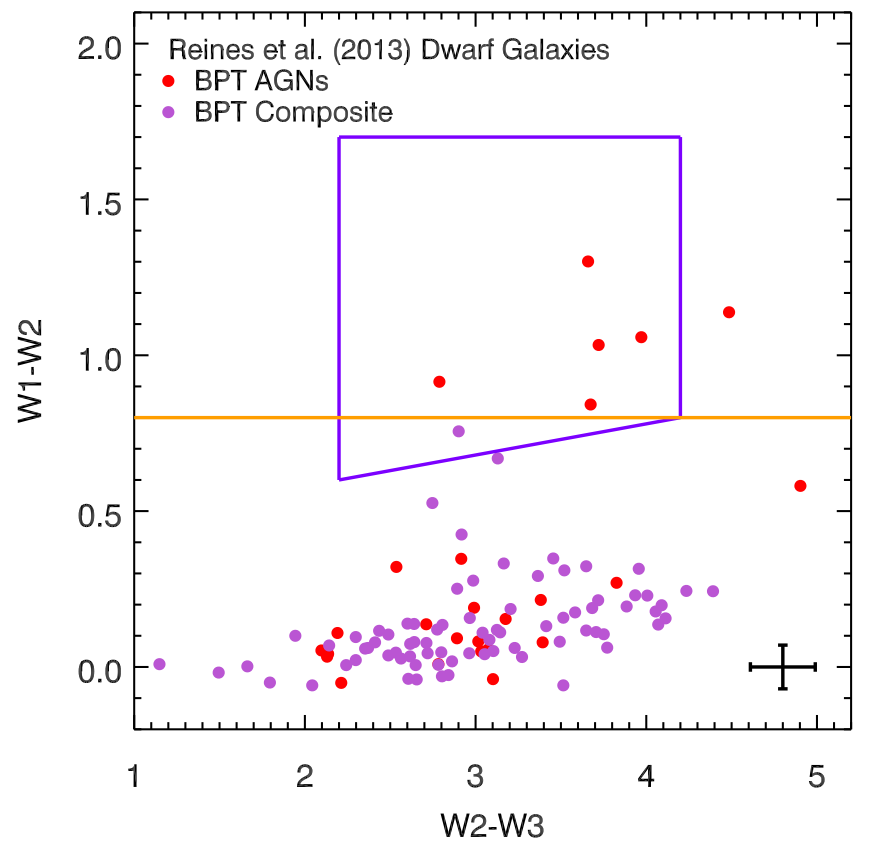

Figure 1. WISE color-color diagram for the dwarf galaxies from Reines et al. (2013) selected as AGN (red points) or composite galaxies (purple points) by their position on the BPT diagram. Four galaxies from the full Reines et al. sample did not have a match to the AllWISE catalog, and 28 galaxies do not have significant detections in the $W 1, W 2$, or $W 3$ bands, as required to place them on this plot. We plot two common AGN selection criteria with different colored lines. In blue, we plot the selection box from Jarrett et al. (2011), and in orange, we plot the $W 1-W 2>0.8$ color criteria from Stern et al. (2012). We also plot the median $W 1-W 2$ and $W 2-W 3$ uncertainties with the error bars in the bottom-right corner.

While we have attempted to account for resolution effects with our usage of the WISE ext_flg value to choose appropriate photometry for each object, we also wish to highlight some systematic effects that could affect our results. First, the Wl, $W 2$, and $W 3$ bands have angular resolutions of 6!" 1,6 ." 4 , and $6 ! 5$, respectively (Wright et al. 2010). As a result, for any object that is slightly resolved in $W 1$ but not in $W 2$, the resulting $W 1$ flux would be underestimated, resulting in a redder $W 1-W 2$ color. This effect could potentially move objects into the WISE color space spanned by AGNs. In addition, for objects with existing 2MASS photometry, which are largely well-resolved by WISE, colors estimated using apertures scaled from the 2MASS XSC shape value might be artificially blue as these apertures may contain foreground stars. Furthermore, these apertures may not contain all of the infrared emission from the object, leading to a systematic underprediction of the fluxes (see Cluver et al. 2014 for an examination of this effect). Because of these photometric effects, we have carefully visually examined the objects that have red WISE colors indicative of potential AGN activity, although objects with ext_flg $=5$ represent a small percentage of the total sample.

\section{MID-INFRARED PROPERTIES OF DWARF GALAXIES AND AGN CANDIDATES}

\subsection{Optically Selected AGNs and Composite Galaxies}

We begin our analysis of the IR properties of dwarf galaxies by exploring the IR colors of the AGNs and composite dwarf galaxies selected from Reines et al. (2013). These 136 dwarf galaxies were initially chosen based on their optical spectroscopic properties and specifically their position on the BPT diagram (Baldwin et al. 1981), a common optical emission-line diagnostic diagram that plots the flux ratio of $[\mathrm{O}$ III $] \lambda 5007 / \mathrm{H} \beta$ against $[\mathrm{N} \mathrm{II}] \lambda 6583 / \mathrm{H} \alpha{ }^{7}$ This diagram separates AGNs from star-forming galaxies based on commonly used dividing lines, an empirical line given in Kauffmann et al. (2003), and a theoretical "maximum starburst line" from Kewley et al. (2001). Objects above the Kewley et al. (2001) maximum starburst line on the diagram are often categorized as AGNs, while objects between the Kauffmann et al. (2003) and Kewley et al. (2001) lines are thought to have contributions to their emission-line flux from both star formation and AGN activity, and are known as "composite" objects.

Of the 136 BPT AGNs and composites from Reines et al. (2013), 104 objects have significant WISE detections as described in Section 2 (four objects did not have a match to the AllWISE catalog, and the other 28 objects do not have significant detections in the $W 1, W 2$, or $W 3$ bands). We plot these 104 dwarf galaxies on the infrared AGN diagnostic WISE color-color plot in Figure 1, which compares the $W 1-W 2$ color to the $W 2-W 3$ color. We color the points based on whether they were classified as an optical AGN (red) or composite galaxy (purple). We also plot common infrared AGN selection criteria with colored lines. In blue, we plot the selection box from Jarrett et al. (2011), and in orange, we plot the $W 1-W 2>0.8$ color criteria from Stern et al. (2012). These selection boxes select for luminous quasars by the presence of power-law emission that extends from the $W l$ through W3 bands due to reprocessed emission from the dust torus around the accretion disk feeding the central supermassive black hole. As can be seen from Figure 1, the majority of the dwarf galaxies selected as AGNs and composite objects by their optical emission would not be selected as an IR AGN on this diagram. Only six of the Reines et al. (2013) BPT-AGN dwarf galaxies would be selected as AGN by the Stern et al. (2012) criterion. Five BPT-AGN and one BPT-composite object would be selected as an AGN by the Jarrett et al. (2011) criteria. We plot the SDSS and WISE thumbnail images for these six objects in Figure 2. From this figure, it can be seen that these optically selected AGNs have significant WISE detections out to the longest wavelengths probed by WISE, evidence of an underlying infrared power-law from AGNheated dust. As discussed in the introduction, more luminous AGNs are more likely to be found with WISE selection, as their infrared emission can be observed above the emission from dust heated by host galaxy star formation. This helps to explain the general trends observed in Figure 1, where five out of six of the objects in the Jarrett et al. (2011) selection box are BPT-AGNs.

The majority of both the BPT-AGNs and BPT-composite dwarf galaxies lie below the AGN selection regions in the area occupied by star-forming galaxies. These objects form a sequence that moves from blue $W 1-W 2$ and $W 2-W 3$ colors to the right and toward redder $W 2-W 3$ colors. For these objects, the AGN IR luminosity must be low enough that the reprocessed infrared emission is overwhelmed by the emission from both the galaxy's stellar continuum, the continuum

\footnotetext{
7 Reines et al. (2013) also identified a sample of AGNs with broad $\mathrm{H} \alpha$ emission as potential AGN candidates, but in this section we focus only on those objects in their sample with optical emission-line ratios indicative of AGN activity.
} 

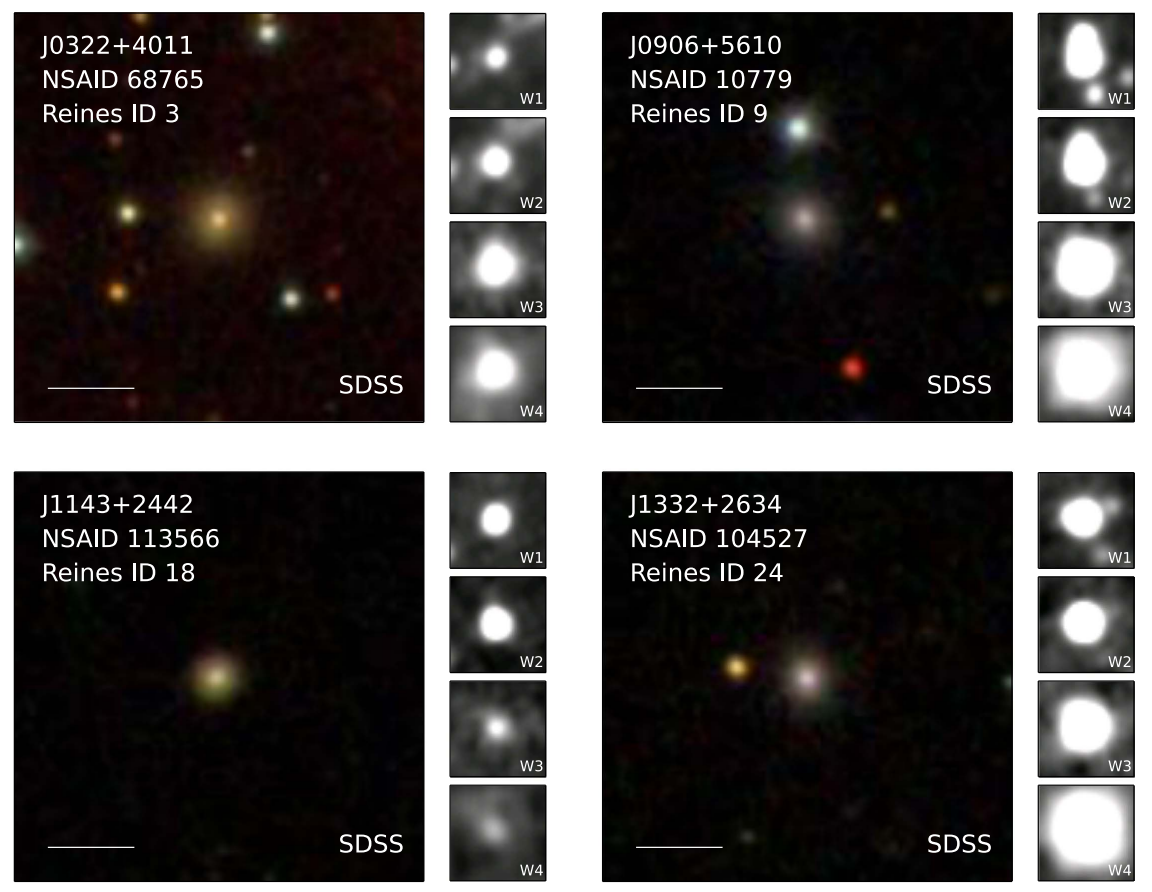

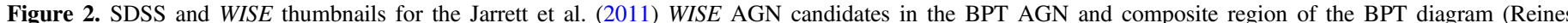

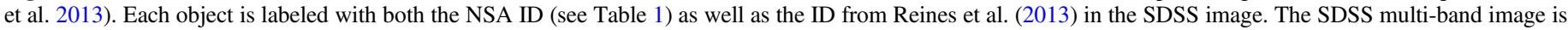

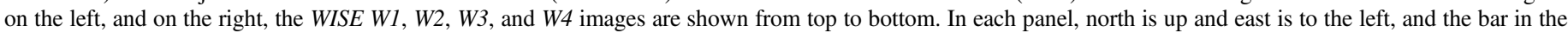

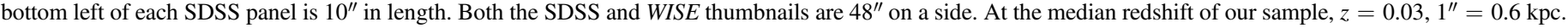

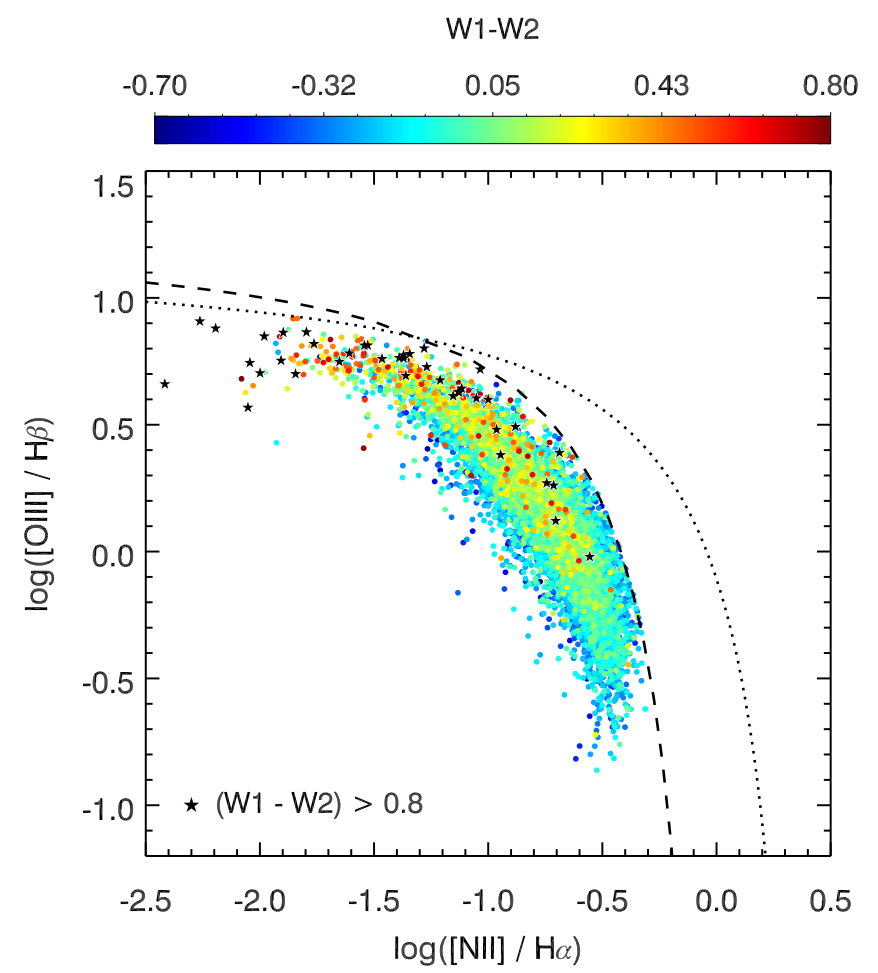

Figure 3. BPT ionization diagram for the star-forming dwarf galaxies described in Section 3.2. The points are colored by their $W 1-W 2$ infrared color, and those objects with $W 1-W 2>0.8$, the criteria used in Stern et al. (2012) for selecting AGN candidates, are plotted with black stars. Overall, there is an observed trend in the figure between the position to the top left of the star-forming sequence and red WISE W1 - W2 color.

emission from dust heated by star formation throughout host galaxy, and polycyclic aromatic hydrocarbon (PAH) emission. We will explore this in more detail in the next section.

\subsection{Optically Selected Star-forming Dwarf Galaxies}

We now look at the dwarf galaxies in the NSA with line ratios dominated by star formation. We use a subsample $(N=14,013)$ of our WISE-selected dwarf galaxies that have significant $(\mathrm{S} / \mathrm{N}>3.0)$ SDSS detections of the strong optical emission lines $(\mathrm{H} \beta,[\mathrm{O} \mathrm{III]} \lambda 5007, \mathrm{H} \alpha$, and $[\mathrm{N} \mathrm{II}] \lambda 6583)$ and fall in the star-forming part of the BPT diagram. We use the wealth of SDSS optical data to understand how dwarf galaxy SF properties relate to WISE infrared colors and explore the potential origin of the red WISE colors observed in some dwarf galaxies. In Figure 3, we show the star-forming subsample on the BPT diagram with points colored by $W 1-W 2$ infrared color. We plot those dwarf galaxies with $W 1-W 2>0.8$, the selection criteria used to select AGNs from Stern et al. (2012), using black stars. For these star-forming dwarf galaxies, we can see that, generally, the $W 1-W 2$ color becomes redder as you move upwards and to the left on the sequence. Star-forming galaxies on the BPT diagram form a sequence in metallicity. Objects with the reddest WISE colors are among the lowest metallicity galaxies in our sample and also have the largest ionization parameters (see Kewley et al. 2013 for a review of how objects move on the star-forming region of the BPT diagram).

\subsubsection{Physical Properties of Star-forming Dwarf Galaxies}

We can use the SDSS spectroscopic data to derive properties of the stellar populations in the dwarf galaxies for comparison to their WISE colors. Some properties, such as galaxy size and optical color, are taken directly from the NSA, but in this section, we outline the methods we use to estimate the host galaxy SFR, stellar age, and ionizing luminosity.

Star Formation Rates. The luminosity of $\mathrm{H} \alpha$ can be used to measure the SFR for a galaxy, following Kennicutt \& Evans 


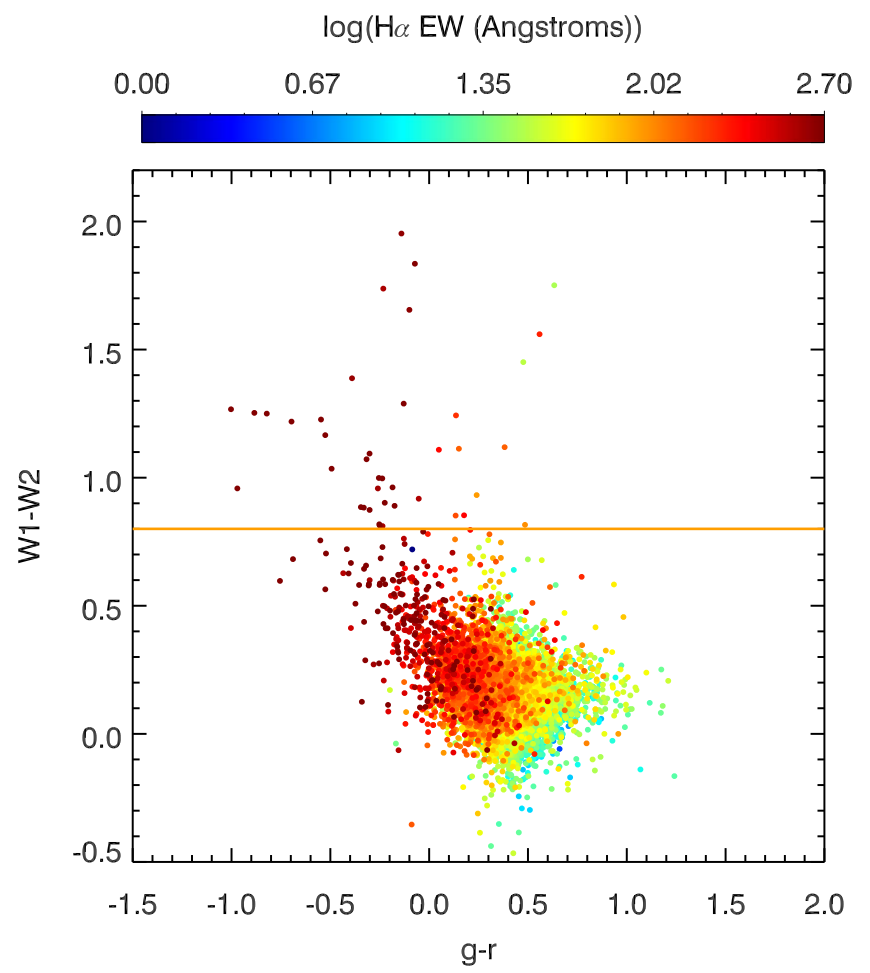

Figure 4. Optical to infrared color for the dwarf galaxies described in Section 3.2. The host galaxies with the reddest WISE colors are, on average, more optically blue, with the highest $\mathrm{H} \alpha$ EWs and youngest ages.

(2012):

$$
\log \left(\operatorname{SFR}\left[M_{\odot} \mathrm{yr}^{-1}\right]\right)=\log \left(L(\mathrm{H} \alpha)\left[\mathrm{erg} \mathrm{s}^{-1}\right]\right)-41.27 .
$$

We have corrected the $\mathrm{H} \alpha$ luminosity for the effects of extinction, using an intrinsic Balmer ratio of $F_{\mathrm{H} \alpha} / F_{\mathrm{H} \beta}=2.86$, $R_{V}=3.1$, and the O'Donnell (1994) extinction curve. This method of estimating extinction is more valid in regions of low obscuration, and is not reliable for the most obscured regions. We also calculate the sSFR, the star formation rate divided by the stellar mass of a galaxy. Since the $\mathrm{H} \alpha$ emission-line fluxes were measured using $3^{\prime \prime}$ diameter fibers, ${ }^{8}$ we calculated the sSFR for each galaxy using masses estimated from the fiberflux values given in the NSA. These fluxes, which were measured within a $3^{\prime \prime}$ aperture, were converted to stellar mass using the method discussed in Reines \& Volonteri (2015), where we used the color-dependent mass-to-light ratio from Zibetti et al. (2009), adopting a solar absolute $i$-band magnitude of $4.56 \mathrm{mag}$ (Bell et al. 2003). While most of the sources have sSFR $<1.0 \mathrm{Gyr}^{-1}$, the majority of the red WISE sources have sSFR $>1.3 \mathrm{Gyr}^{-1}$. In addition, we also use the Petrosian halflight radius from the NSA to estimate the SFR surface density $\left(\Sigma_{\mathrm{SFR}}\right)$, the SFR divided by the area of the galaxy in $\mathrm{kpc}^{2}$.

Stellar Age. The equivalent width (EW) of the hydrogen Balmer lines anticorrelates with the age of a stellar population (Stasińska \& Leitherer 1996). We estimate stellar ages by comparing measured $\mathrm{H} \alpha$ values to Starburst99 models (Leitherer et al. 1999), assuming a Salpeter (1955) IMF with power-law $\alpha=2.35$ and a metallicity of $Z=0.004$ (where

\footnotetext{
8 The $\mathrm{H} \alpha$ flux and EW estimates we use in this work are measured from SDSS fiber measurements and are dependent on the exact morphology and redshifts of the galaxies. As a result, we may be missing $\mathrm{H} \alpha$ flux from star formation outside the fiber aperture. The majority of those objects with red WISE colors are smaller than the SDSS aperture.
}

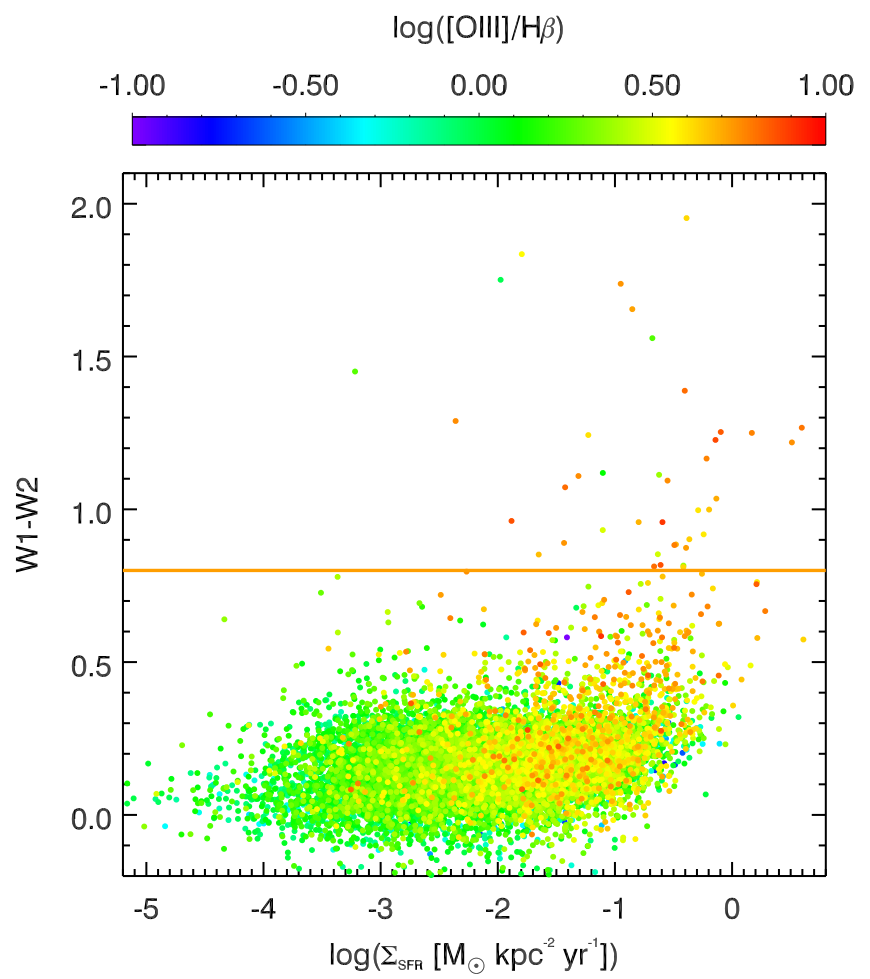

Figure 5. WISE W1 - W2 color plotted against the SFR surface density $\left(\Sigma_{\mathrm{SFR}}\right)$ for the dwarf galaxies described in Section 3.2, with the points colored by the $[\mathrm{O} \mathrm{III}] / \mathrm{H} \beta$ line ratio. Those galaxies with the reddest $W 1-W 2$ colors have the highest SFR surface densities.

$Z=0.02$ represents solar metallicity). The majority of the galaxies with red WISE colors have high $\mathrm{H} \alpha$ EWs and inferred stellar ages less than $7 \mathrm{Myr}$.

Ionizing Luminosity. We use the extinction-corrected $\mathrm{H} \alpha$ luminosity to estimate the rate of ionizing photons $\left(Q_{\mathrm{Lyc}}\right)$ for these objects following the procedure from Reines et al. (2008):

$$
\left(\frac{Q_{\mathrm{Lyc}}}{\mathrm{s}^{-1}}\right) \geqslant 2.25 \times 10^{12}\left(\frac{T_{e}}{10^{4} \mathrm{~K}}\right)^{0.07}\left(\frac{L_{\mathrm{H} \alpha} / 2.86}{\mathrm{erg} \mathrm{s}^{-1}}\right) .
$$

Here, we start from Equations (2) and (3) from Condon (1992), and then assume Case $\mathrm{B}$ recombination, where $F_{\mathrm{H} \alpha} / F_{\mathrm{H} \beta}=2.86$. We further assume an electron temperature of 10,000 K (Izotov et al. 1997), and, as seen from the above equation, our estimated ionizing photon rate is only a lower limit due to the fraction of ionizing radiation that is absorbed by dust or escapes the starforming regions before ionizing hydrogen atoms. The dwarf galaxies in our sample with the reddest $W 1-W 2$ colors have large $\mathrm{H} \alpha$ luminosities and values of $Q_{\mathrm{Lyc}}$ equivalent to greater than several thousand O-type stars.

\subsubsection{Mid-IR Colors of Star-forming Dwarf Galaxies}

To investigate the origin of the infrared emission from the star-forming dwarfs, we plot the optical $g-r$ and infrared $W 1-W 2$ colors for these objects in Figure 4. Here, we can see that the galaxies with the bluest optical colors have the reddest infrared colors. We have colored the points by the EW of the $\mathrm{H} \alpha$ emission line taken from the NSA, and we see that those dwarf galaxies with the reddest WISE colors have the highest $\mathrm{H} \alpha$ EW. We plot WISE W1 - W2 color as a function of $\Sigma_{\mathrm{SFR}}$ for each galaxy in Figure 5, and we see that the 

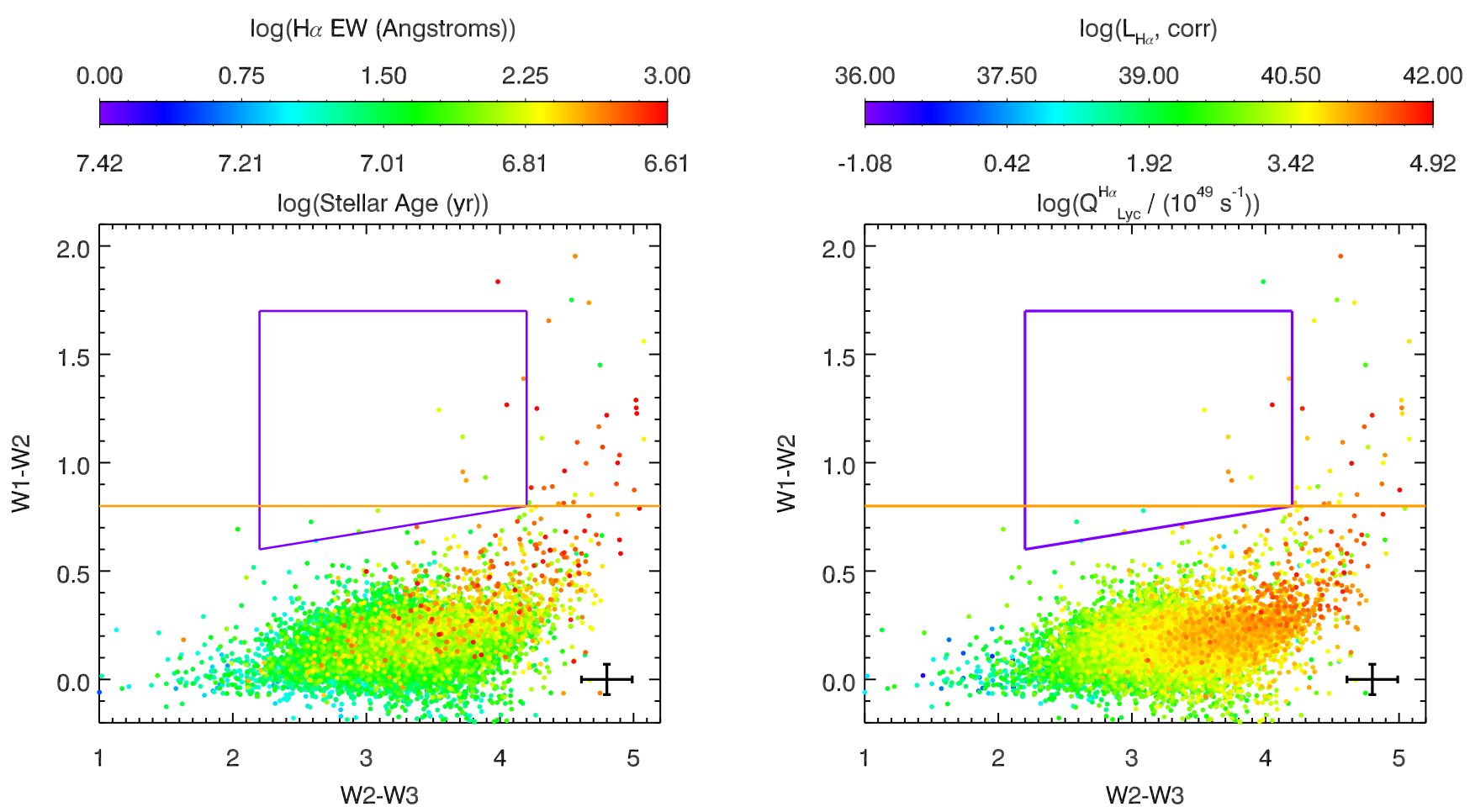

Figure 6. (Left) WISE color-color diagram for the star-forming dwarf galaxies in our sample, colored by the EW of the H $\alpha$ emission line. The color bar indicates the derived ages of the stellar populations estimated from the H $\alpha$ EW and Starburst99 models. (Right) WISE color-color diagram for the star-forming objects in our sample, colored by the extinction-corrected luminosity of the $\mathrm{H} \alpha$ emission line. The color bar also provides an estimate for the rate of ionizing photons $\left(Q_{\mathrm{Lyc}}\right)$ for these objects, as described in Equation (2). We also plot the median $W 1-W 2$ and $W 2-W 3$ uncertainties with the error bars in the bottom-right corner of each panel.

majority of the objects with the reddest $W 1-W 2$ colors also have the highest $\Sigma_{\mathrm{SFR}}$, further supporting the idea that we are observing compact young starbursts in these dwarf galaxies with red WISE colors. We note that our usage of aperture fluxes to derive SFRs would lead to values of $\Sigma_{\text {SFR }}$ for objects with Petrosian half-light radii larger than the SDSS fiber to be underestimated, although this effect does not significantly change the results seen in Figure 5.

We show WISE color-color diagrams for the dwarf starforming galaxies in Figure 6. We color the points based on the EW (left panel) and luminosity (right panel) of the $\mathrm{H} \alpha$ emission line. In both panels of Figure 6, we also plot the same AGN selection boxes shown in Figure 1. We can see that the majority of the objects lie outside the Jarrett et al. (2011) selection box. At the reddest $W 2-W 3$ colors, the star-forming sequence extends upwards to redder $W 1-W 2$ colors, wrapping around the Jarrett et al. (2011) AGN selection box and above the Stern et al. (2012) selection line. In the left panel of Figure 6, we see that those objects with the reddest WISE infrared colors have the largest $\mathrm{H} \alpha \mathrm{EW}$ values and youngest stellar ages, also indicated in Figure 4. Similarly, in the right panel of Figure 6, we can see an even stronger trend for the objects with the reddest WISE colors to have the largest $\mathrm{H} \alpha$ luminosities and rate of ionizing photons.

We explore the dwarf galaxy sequence on the WISE colorcolor diagram as a function of dust temperature in Figure 7. Here, on the left, we plot the same dwarf galaxies and AGN selection boxes as in Figure 6, but now the objects are colored by their sSFR. We see that the sequence of BPT-selected starforming dwarf galaxies drives to the right and upward, and is correlated with the sSFR. We also plot extreme WISE dwarf galaxies from Griffith et al. (2011) and Izotov et al. (2011), which have $W 1-W 2 \sim 2.0$. These rare objects are at the extreme end of the sequence traced by the star-forming dwarf galaxies in our sample. We also plot the WISE colors for the infrared-bright star-forming galaxies Arp220 and M82, as measured from their SWIRE SED templates (Polletta et al. 2007). Finally, in this panel we plot color tracks that we generated by linearly combining an elliptical galaxy template from Assef et al. (2010) with a simple singletemperature blackbody (BB) at $z=0$. For these tracks, we chose an elliptical galaxy template as this template does not show evidence for dust in the infrared. We plot three tracks at three different dust temperatures, $400 \mathrm{~K}$ (blue), $330 \mathrm{~K}$ (olive), and $300 \mathrm{~K}$ (magenta). The position along the track (to the right and upwards) reflects the relative normalization of the $\mathrm{BB}$ and the galaxy template, which we parameterize using $f_{\mathrm{BB}, 10 \mu \mathrm{m}}$, the fraction of the total flux emitted from the BB at $10 \mu \mathrm{m}$. In the right panels we show example SEDs for both the $300 \mathrm{~K}$ (top) and $400 \mathrm{~K}$ (bottom) examples, with the galaxy template in blue, the BB in red, and the total flux in green. We overplot the $W 1$, $W 2$, and $W 3$ fluxes, and provide the $W 1-W 2$ and $W 2-W 3$ colors in each panel.

From the tracks and SEDs in Figure 7, we can see how the sequence of dwarf galaxies arises in WISE color space. At a fixed BB normalization, as the temperature of the hottest dust in a given galaxy increases, galaxies move upward and to the left, satisfying the Stern et al. (2012), and potentially the Jarrett et al. (2011), AGN selection criteria. Alternately, at a fixed BB temperature, as the normalization of the BB template increases, objects move upward and to the right. While a BB is a simplistic approximation of the dust emission observed in the infrared, our model does replicate the observed range in the $W 1-W 2$ and $W 2-W 3$ colors for the dwarf galaxies. We emphasize that our model lacks PAH emission, which may significantly change the observed colors, although lower PAH 

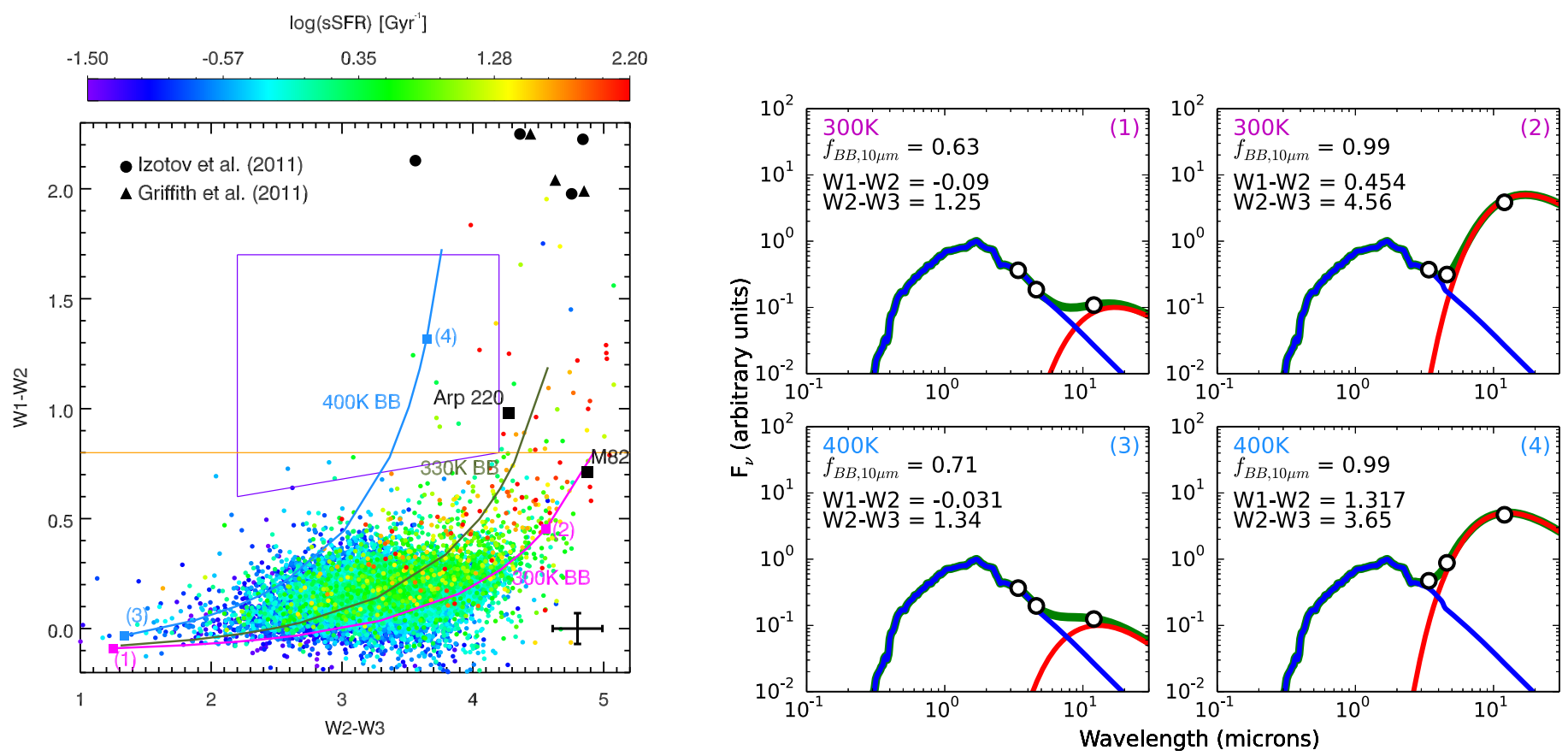

Figure 7. WISE color-color diagram for the BPT-selected star-forming dwarf galaxies. The objects are colored by sSFR as shown in the color bar above the figure. Those objects with the highest sSFR values are found to the right and upwards on the diagram. We also plot two samples of objects with extreme WISE W1 - W2 colors from Izotov et al. (2011) and Griffith et al. (2011), which appear to be a natural extension of the sequence of dwarf galaxies that extends from the bottom left corner to the top right corner. On top of the sequence, we plot color tracks generated by adding a blackbody to an Assef et al. (2010) elliptical galaxy template (an elliptical galaxy template was chosen as the IR emission in this template is lacking in dust emission, which we model with the blackbody) at $z=0$. The different colors (magenta is $300 \mathrm{~K}$, olive is $330 \mathrm{~K}$, and blue is $400 \mathrm{~K}$ ) represent different blackbody temperatures, and the sequence is generated by varying the ratio of the total IR luminosity of the blackbody with respect to the elliptical template, as shown in the sample SEDs plotted on the right panels. The colored numbers in the top right of each panel correspond to the positions on the left color-color diagram. In each panel on the right we also provide $f_{\mathrm{BB}, 10 \mu \mathrm{m}}$, the fraction of the total flux that is emitted by the BB at $10 \mu \mathrm{m}$. The dwarf galaxies seem to trace a sequence in color space represented by more luminous dust emission, with a scatter in the sequence that arises due to different maximum dust temperatures. We also plot the median $W 1-W 2$ and $W 2-W 3$ uncertainties with the error bars in the bottom-right corner of the left panel.

abundance has been found in low-metallicity, high-sSFR dwarf galaxies (Wu et al. 2005; Madden et al. 2006; Engelbracht et al. 2008; Galliano et al. 2008; Rémy-Ruyer et al. 2015). The BB temperatures from our models are consistent with those estimated for dust heated from star formation in dwarf galaxies in the literature (Johnson et al. 2004; Engelbracht et al. 2005; Jackson et al. 2006; Reines et al. 2008). In addition, as the WISE wavelength coverage does not extend to the far-infrared, we cannot quantify cooler dust in these galaxies. We further discuss the implications of these results in Section 4.

\subsubsection{Mid-IR-selected AGN Candidates}

While our results indicate that the majority of star-forming dwarf galaxies lie outside of the commonly used WISE AGN selection boxes, there are potential IR-selected AGN candidates that we want to highlight. We find only 10 dwarf star-forming galaxies with WISE colors that put them in the Jarrett et al. (2011) AGN selection box. We present SDSS thumbnails for these objects in Figure 8. These objects span a range in optical color, but are on average bluer than the full sample of dwarf galaxies (e.g., see Figure 4). Three out of the ten objects do not have significant ( $\mathrm{S} / \mathrm{N}>3$ ) W4 photometry (NSA ID 93798, 109919, and 117162), as would be expected for an AGN. Two of these objects, NSA ID 109919 and 93798, have low surface brightness, and do not show strong photometric evidence for a central nuclear source. NSA 93978 was presented by Secrest et al. (2015) who demonstrated that this galaxy was detected by XMM-Newton with a luminosity of $L_{2-10 \mathrm{keV}}=2.4 \times 10^{40} \mathrm{erg} \mathrm{s}^{-1}$. We further discuss this object in Section 3.4. For the remainder of the objects, their morphologies are either compact blue nuggets (i.e., 98135), objects with blue cores and extended features (4610, and $118025)$, or objects with evidence for disks and nuclear cores (6205, 57649, and 151888).

\subsection{Galaxies Without Optical Emission-line Flux Measurements}

There are a number of dwarf galaxies in our sample (4469 objects) with low signal-to-noise ratio $(\mathrm{S} / \mathrm{N})$ detections of the emission lines in existing SDSS spectra or no spectroscopic information in the NSA. From this subsample, we initially found 68 objects that fall into the Jarrett et al. (2011) selection box. We examined these objects to explore whether they had any alternate spectroscopic information: many have SDSS DR12 spectra that allowed us to determine that the NSA redshifts (and therefore masses) were incorrect for these objects, and they are likely unobscured quasars at $z \sim 1-2$. There are two objects, NSA IDs 110100 and 172349, that appear to be a chance alignment of a dwarf galaxy and a background quasar. After removing any quasar contaminants, we are left with only 25 potential IR-selected AGN dwarf galaxy candidates, and we show their SDSS and WISE thumbnails in Figures 10-12 in the Appendix. We note that two of these objects, NSA IDs 135305 and 150927, have poor W3 photometric fits (for these objects, the WISE w3rchi2 value, 

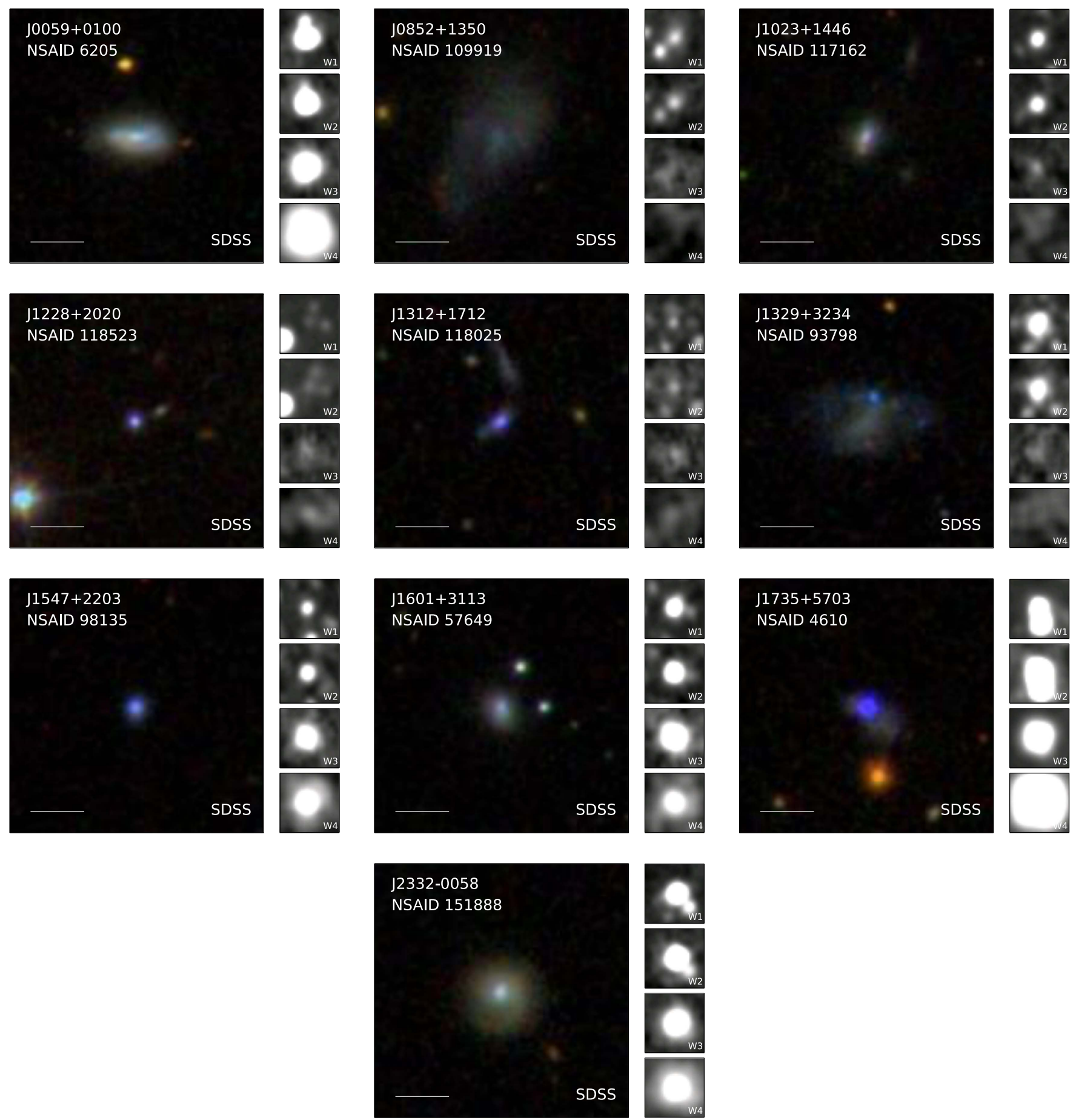

Figure 8. SDSS and WISE thumbnails for the WISE-selected (Jarrett et al. 2011) AGN candidates in the star-forming region of the BPT diagram. In each panel, north is up and east is to the left, and the bar in the bottom left of each SDSS panel is $10^{\prime \prime}$ in length. Both the SDSS and WISE thumbnails are 48" on a side. At the median redshift of our sample, $z=0.03,1^{\prime \prime}=0.6 \mathrm{kpc}$.

the $\chi^{2}$ of the $W 3$ profile fitting, is W3rchi2 $\geqslant 2$ ), and their $W 3$ flux might be underpredicted, leading to these objects being bluer in the $W 2-W 3$ color. Many of the dwarf galaxies do not have significant $W 4$ detections. We would expect $W 4$ detections from the power-law emission typically associated with an AGN, as seen for the BPT-AGNs in Figure 2. While the sizes and $g-r$ colors for these objects span a similar range as what was measured for the full sample of BPT star-forming objects in Figures 4 and 5, many are either optically redder or physically larger than the star-forming dwarf galaxies with red
$W 1-W 2$ colors. Optical spectroscopy is necessary to better understand these systems.

We list the NSA ID, SDSS ID, redshift, optical color, and WISE colors for all of the AGN candidates (using the Jarrett et al. 2011 selection box) in Table 1. Furthermore, we plot all the NSA dwarf galaxies that have WISE colors placing them inside the Jarrett et al. (2011) selection box in Figure 9, with the points colored by their optical properties. We note that these objects do not span the entire Jarrett et al. (2011) selection space, but cluster near the right and bottom of the box. 


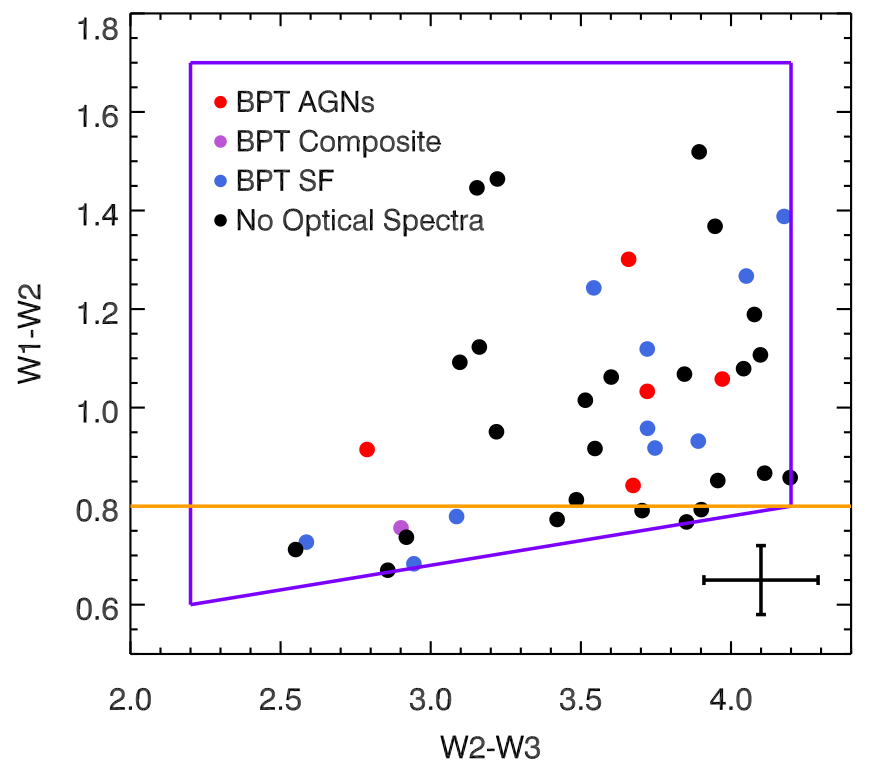

Figure 9. WISE color-color diagram focusing on those objects that fall in the Jarrett et al. (2011) selection box. We plot the positions of the Reines et al. (2013) dwarf AGNs in red, dwarf composite objects in purple, the BPT SF objects in blue, and the galaxies without SDSS spectroscopic information in black. We also plot the median $W 1-W 2$ and $W 2-W 3$ uncertainties with the error bars in the bottom-right corner.

\subsection{Comparison to Recent Searches for AGNs in Dwarf Galaxies Using Mid-IR Colors}

Recently, Satyapal et al. (2014) and Sartori et al. (2015) used WISE data to search for the presence of AGNs in dwarf and bulgeless galaxies. These objects were used to draw conclusions about the AGN population that would be missed in optical and X-ray surveys. Of the 30 AGN candidates using the Jarrett et al. (2011) selection box and presented in Satyapal et al. (2014), only one object is in the mass and redshift range probed by our analysis here (the others are either at higher masses or higher redshifts), and it is found in our sample of star-forming dwarfs in the Jarrett et al. (2011) box (NSA ID 93798). Secrest et al. (2015) measured an X-ray luminosity from XMM-Newton observations (extracted with a $\sim 15^{\prime \prime}$ aperture) of $L_{2-10 \mathrm{keV}}=2$. $4 \times 10^{40} \mathrm{erg} \mathrm{s}^{-1}$ for this object, and claim that the W1 - W2 color is strongly indicative of AGN activity. While this object is indeed in the Jarrett et al. (2011) selection box, given the median uncertainties on the WISE photometry, its infrared colors are consistent with this object potentially being scattered from the primary locus of star-forming galaxies and it does not have a strong power law to longer infrared wavelengths as expected for an AGN.

Sartori et al. (2015) presented a sample of 83 objects with photometry placing them in the Jarrett et al. (2011) selection box, using data from the older WISE All-sky data release. These authors only required $\mathrm{S} / \mathrm{N}>2$ for detections in the $W 1$, $W 2$, and $W 3$ photometric bands. We cross-matched against the more up-to-date AllWISE selection, and using a $\mathrm{S} / \mathrm{N}$ cut of 3.0, we find that only 34 objects $(\sim 41 \%)$ continue to fall inside the Jarrett et al. selection box. ${ }^{9}$ Of these 34 objects, only 15 are in our parent sample of dwarf galaxies. The remaining 19 objects are either not in the NSA because their redshifts are greater

\footnotetext{
9 All 83 of the objects in the Sartori et al. (2015) sample have updated AllWISE $W 1$ and $W 2$ photometry with $\mathrm{S} / \mathrm{N}>3.0$, but 26 of the objects have $W 3$ photometry with $\mathrm{S} / \mathrm{N}<3$, only 10 of which have $2<\mathrm{S} / \mathrm{N}<3$.
}

than 0.055 , or are in the NSA but not classified as dwarf galaxies by our mass criterion. Looking closer at these 15 dwarf galaxies, 10 are found in Table 1 (NSA IDs 4610, 6205, $10311,53934,57649,93798,109919,117162,118025$, and 118523), 4 of the 15 are BPT-selected AGNs or composite galaxies (NSA IDs 10779, 79874, 124050, and 125318), and one is the previously discussed quasar contaminant (NSA ID 110100). One object that was listed as a Stern et al. (2012) candidate but not a Jarrett et al. (2011) candidate in Sartori et al. (2015) moved into the Jarrett et al. (2011) selection box with the updated AllWISE photometry (NSA ID 98135), and one object we have listed in Table 1 does not appear in Sartori et al. (2015) (NSA ID 151888).

\subsection{The Feasibility of Detecting Supermassive Black Holes in Dwarf Galaxies with WISE}

Finding evidence for AGNs in dwarf galaxies is challenging as AGNs at a given accretion rate have lower bolometric luminosities due to the smaller masses of the central black holes, making them harder to observe above the emission due to star formation in their host galaxies. It has been shown using common emission-line AGN selection techniques that there is a strong bias against finding AGNs in low-mass galaxies for this reason (Aird et al. 2012; Bongiorno et al. 2012; Hainline et al. 2012; Trump et al. 2015). To that end, we sought to explore the range of black hole masses in potential AGNs in dwarf galaxies that are detectable using WISE colors, following a similar discussion in Reines et al. (2013).

We calculate the minimum black hole detectable in the scenario where the AGN flux must be above some median flux from star formation at 3-4 $\mu \mathrm{m}$. Assuming that the bulk of the objects with $W 1-W 2<0.5$ have WISE fluxes that are dominated by dust heated from star formation, we calculate the median $W 1$ and $W 2$ fluxes for all of these objects after shifting them to the median redshift of our sample. We do not perform this calculation using the $W 3$ fluxes, as the differences in cold dust temperatures across the sample is more obvious at $12 \mu \mathrm{m}$ than at the shorter wavelengths. We calculate median $W 1$ and $W 2$ fluxes of 382 and $243 \mu \mathrm{Jy}$ respectively. From here, we use the low resolution AGN spectral template from Assef et al. (2010) to estimate the AGN bolometric luminosity for an object at the median redshift of $z \sim 0.03$ given these flux limits. We note that this template was derived using more luminous AGNs, and the actual relationship between the IR and bolometric luminosities may vary for lower-mass black holes. We derive minimum black hole masses of $(1-3) \times 10^{3} M_{\odot}$, if we assume that the black hole is accreting at the Eddington limit, where $L_{\mathrm{Edd}}=1.3 \times 10^{38}\left(M_{\mathrm{BH}} / M_{\odot}\right) \mathrm{erg} \mathrm{s}^{-1}$. However, black hole accretion at such high levels is quite rare (Schulze \& Wisotzki 2010), and we also calculate that a black hole with mass $10^{5} M_{\odot}$ (similar to the masses of the BHs measured in dwarf galaxies in Reines et al. 2013; Baldassare et al. 2015) would only need to radiate at $1 \%-6 \%$ of its Eddington luminosity to be observed given the median flux from star formation in our sample.

\section{DISCUSSION AND CONCLUSIONS}

To better understand the birth and growth of supermassive black holes, it is fundamentally important to find evidence for AGN activity in low-mass dwarf galaxies. Here, we looked at the mid-IR properties of a sample of dwarf galaxies at 

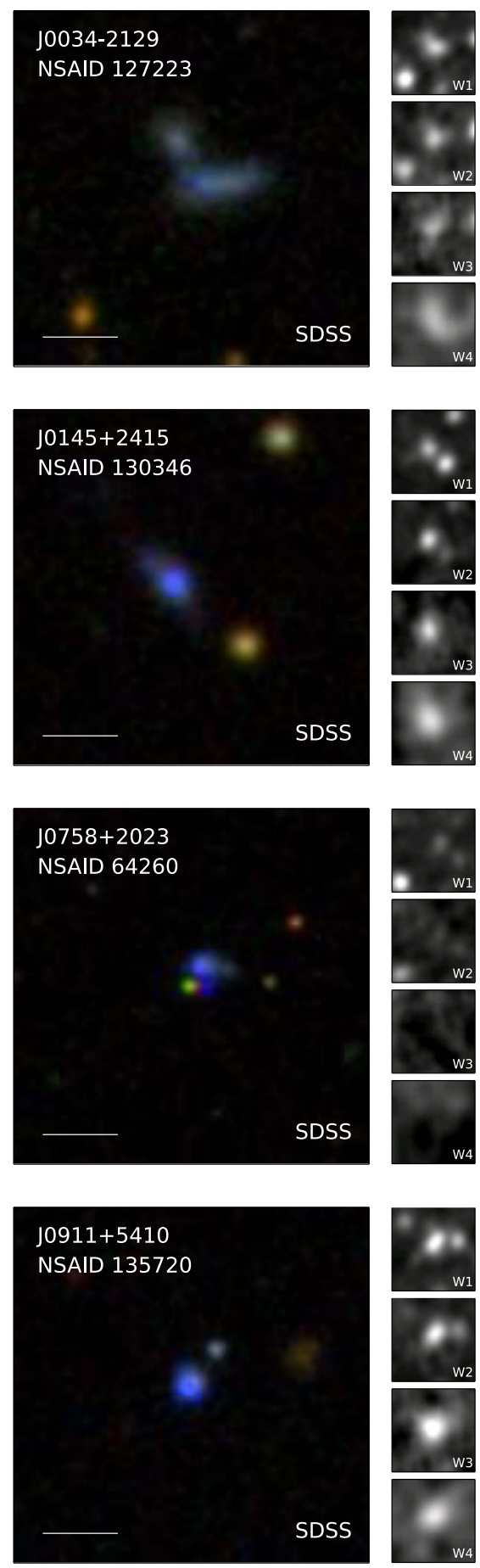
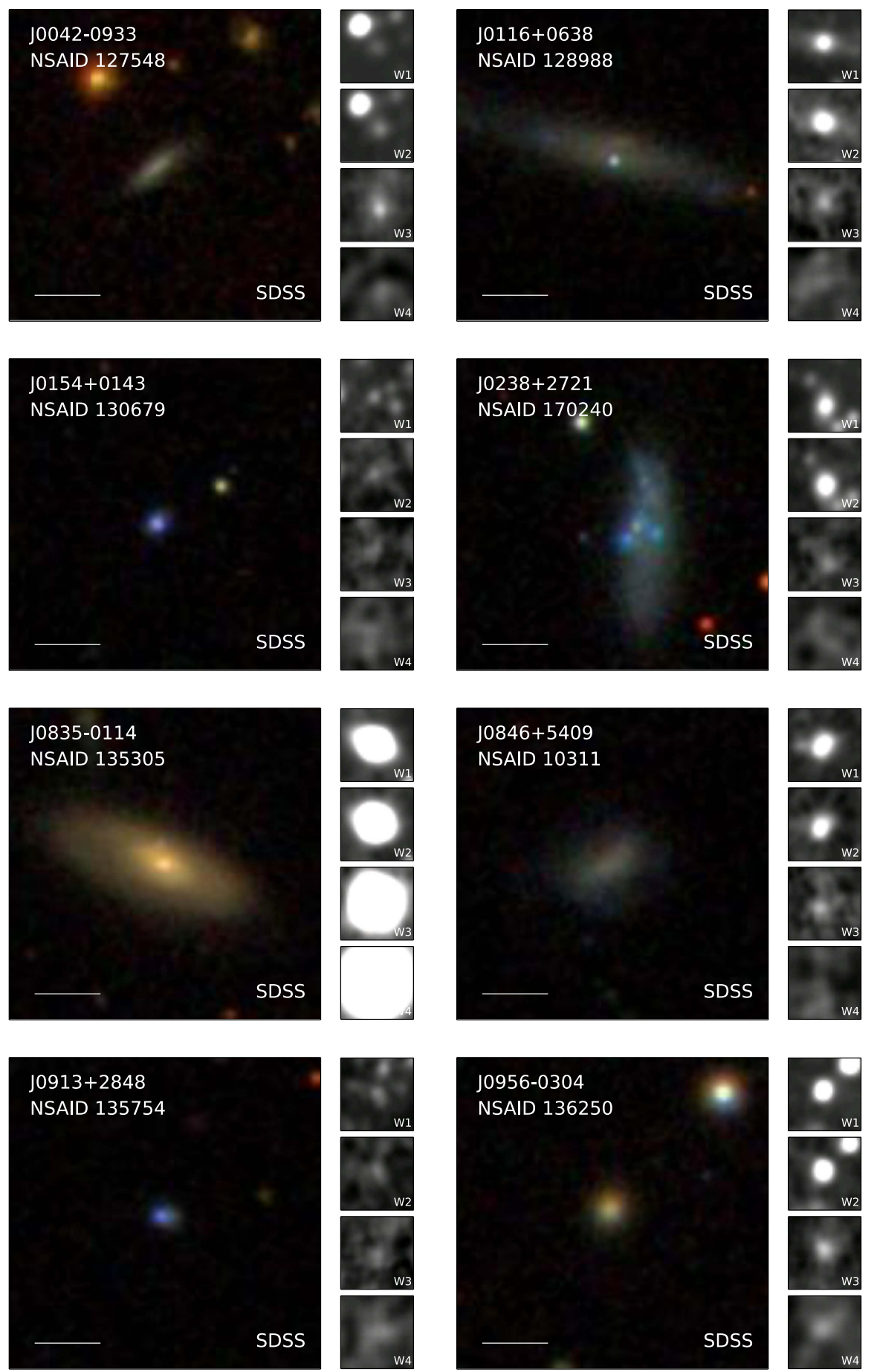

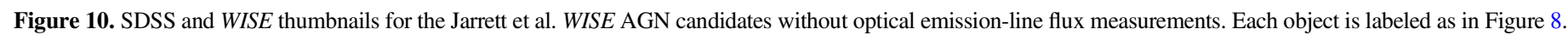

$z<0.055$ to explore the use of WISE colors to select for AGN activity. We used the most up-to-date AllWISE photometry and individually inspected each candidate AGN to select a sample that is significantly smaller than previous samples of IRselected AGNs in dwarf galaxies.

Our main conclusions are:

1. The majority of optically selected AGNs in dwarf galaxies have IR colors that are dominated by their host galaxies.

2. Dwarf galaxies with the reddest WISE colors are compact, blue galaxies with young stellar ages and high sSFRs.
Dwarf galaxies with extreme star formation are capable of heating dust to temperatures producing $W 1-W 2>0.8$, (e.g., Stern et al. 2012), and this single color cut alone should not be used to select AGNs in dwarf galaxies.

3. We provide a sample of 41 dwarf galaxies in the NSA that have WISE colors in the Jarrett et al. (2011) AGN selection box, 6 of which have optical spectroscopic evidence for an AGN (Reines et al. 2013). While the majority of the objects in our sample have been included in previous samples of WISE-selected dwarf galaxy AGN candidates, our sample is much smaller due to the updated WISE photometry, more conservative selection 

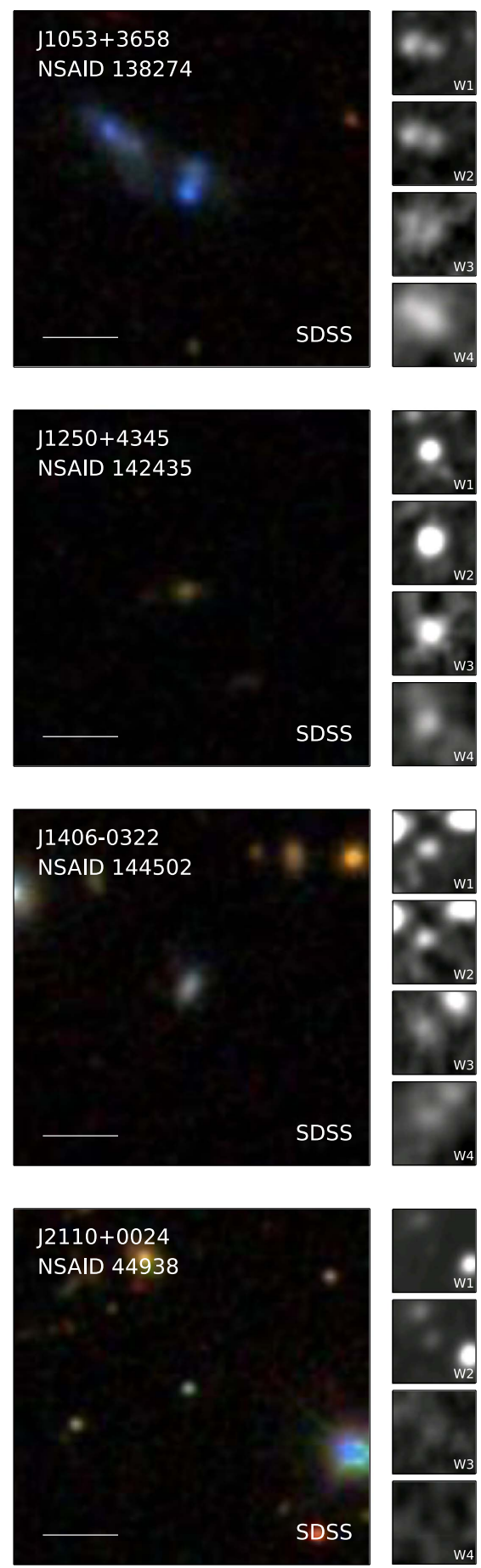
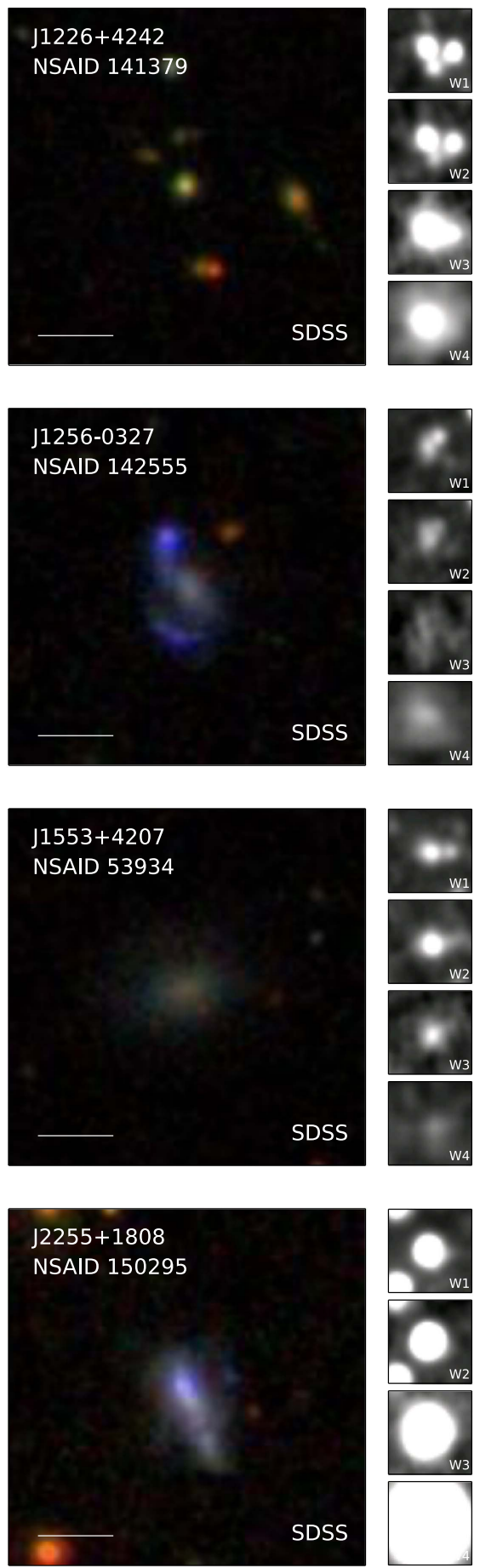
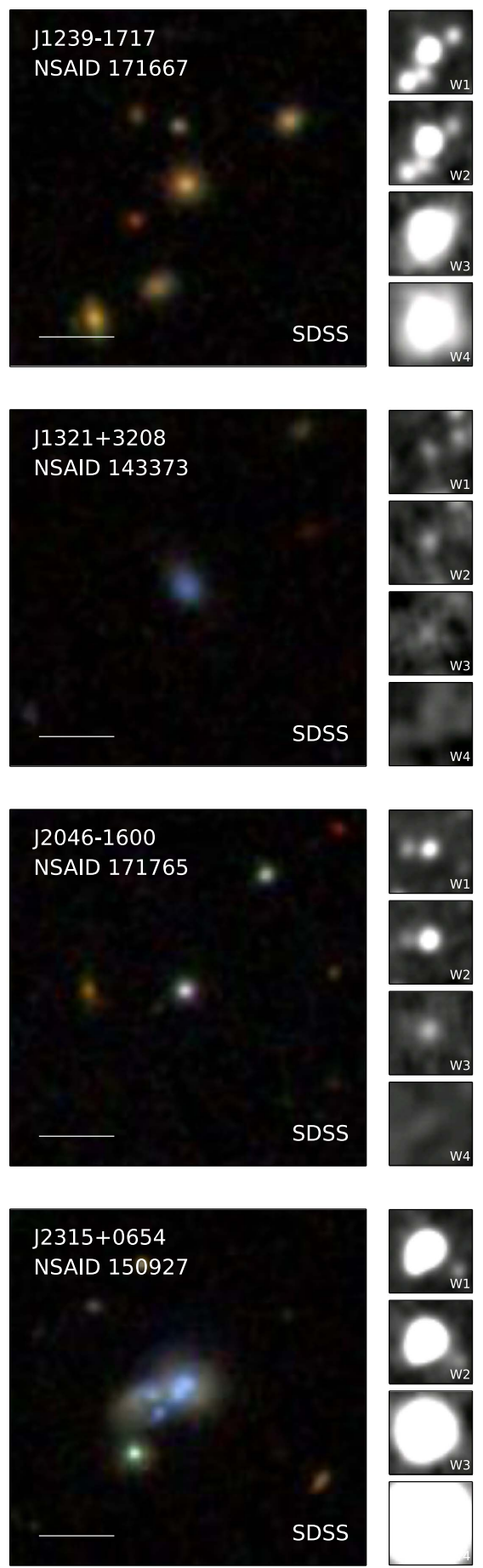

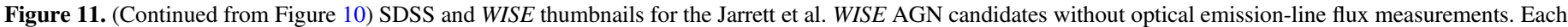
object is labeled as in Figure 8.

criteria and $\mathrm{S} / \mathrm{N}$ thresholds, and the removal of spurious candidates. We caution that follow-up observations are necessary to confirm the presence of active massive black holes in the other 35 objects.

From our analysis, optically blue, high-sSFR dwarfs with young starbursts and associated high ionizing fluxes can have red mid-IR colors that could be mistaken for AGN activity, particularly if using a simple $W 1-W 2$ color cut. While these objects have a population of young stars that can heat dust to temperatures that result in red $W 1-W 2$ colors
$(W 1-W 2>0.5-0.6)$, they primarily have very red $W 2-W 3$ colors $(W 2-W 3>4.2)$, which is less extreme, but similar to the $W 2-W 3$ colors seen for the dwarf star-forming galaxies from Izotov et al. (2011).

AGNs, which can heat dust to even higher temperatures, are predominantly found in a different region of WISE color-color space, and we only find 10 optically selected star-forming dwarf galaxies that would be classified as an AGN by the Jarrett et al. (2011) selection criteria. Of those objects, only 5 have strong W3 and W4 fluxes as would be expected for an 
Table 1

WISE-selected/Jarrett et al. (2011) AGN Candidates

\begin{tabular}{lccccc}
\hline \hline NSA ID & SDSS ID & $z$ & $g-r$ & $W 1$ & $W 1-W 2$ \\
\hline \multicolumn{3}{c}{ BPT-selected AGNs $^{\mathrm{a}}$} \\
\hline 68765 & $\mathrm{~J} 032224.64+401119.8$ & 0.026 & 0.618 & $14.012 \pm 0.020$ & 0.842 \\
10779 & $\mathrm{~J} 090613.75+561015.5$ & 0.047 & 0.426 & $13.400 \pm 0.024$ & 1.301 \\
125318 & $\mathrm{~J} 095418.15+471725.1$ & 0.033 & 0.448 & $13.859 \pm 0.027$ & 1.058 \\
113566 & $\mathrm{~J} 114359.58+244251.7$ & 0.050 & 0.683 & $14.275 \pm 0.028$ & 0.915 \\
104527 & $\mathrm{~J} 133245.62+263449.3$ & 0.047 & 0.281 & $13.514 \pm 0.025$ & 3.659 \\
& & & & & \\
& & & & & \\
\end{tabular}

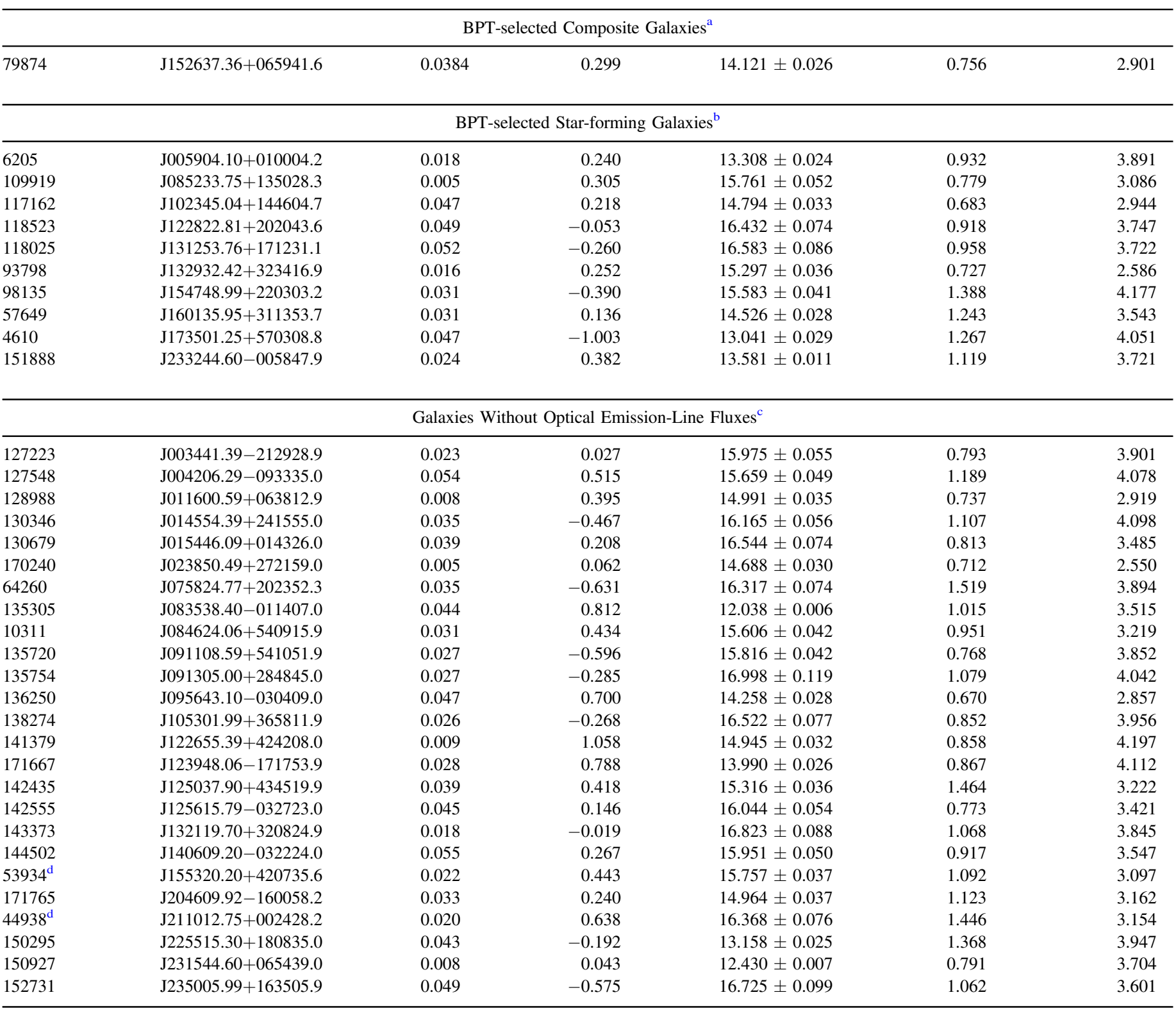

Notes.

a Objects from Reines et al. (2013). The SDSS and WISE thumbnails for these objects are shown in Figure 2.

b The SDSS and WISE thumbnails for these objects are shown in Figure 8.

c The SDSS and WISE thumbnails for these objects are shown in Figures 10-12 in the Appendix.

$\mathrm{d}$ These objects have SDSS spectra, but one or more of the emission lines used for BPT selection lack sufficient S/N for our optical classification.

AGN. In addition, these objects are consistent with the overall trend of star-forming galaxies in WISE color space, and these objects could represent an extreme star-forming population that has scattered into the Jarrett et al. (2011) selection box. We also found 25 additional dwarf galaxies that fell into the Jarrett et al. (2011) box that do not have optical spectroscopy, or their SDSS emission lines were not strong enough to classify them on the BPT diagram. We include them as candidates, but we 


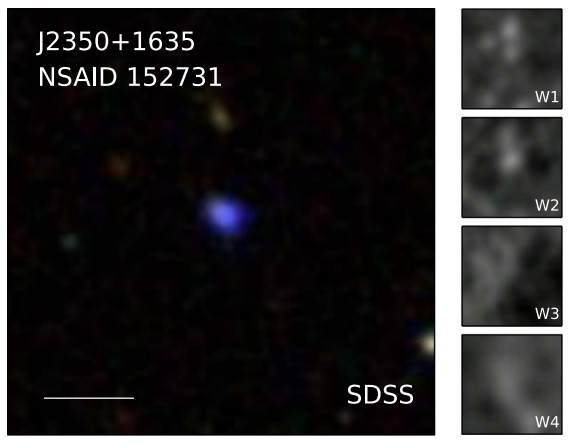

Figure 12. (Continued from Figure 10) SDSS and WISE thumbnails for the Jarrett et al. WISE AGN candidates without optical emission-line flux measurements. Each object is labeled as in Figure 8.

caution against using the mid-IR colors of these objects alone to classify them as AGNs; follow-up observations at optical or $\mathrm{X}$-ray wavelengths would be helpful to understand the nature of their IR emission.

Overall, it is important to use both $W 1-W 2$ and $W 2-W 3$ colors when selecting candidate AGNs in dwarf galaxies, as any selection of AGNs in dwarf galaxies that only uses $W 1-W 2$ color (for instance, the selection method of Stern et al. 2012, which has been demonstrated to be reliable at higher masses) will include a large amount of contamination from dwarf star-forming galaxies. Starting with the BPT starforming galaxies discussed in Section 3.2, we find that 42 dwarf galaxies have $W 1-W 2>0.8$ (compared to only 10 in the Jarrett et al. 2011 selection box), while 183 objects have $W 1-W 2>0.5$. This contamination from star-forming dwarf galaxies is likely the cause of the observed rise in the fraction of AGN candidates at lower galaxy masses in Satyapal et al. (2014) and Sartori et al. (2015). While we certainly cannot rule out the presence of AGNs in the optically selected star-forming dwarf galaxies with red WISE colors, the systematic correlations between star formation properties and infrared colors lead us to conclude that the infrared emission is unlikely to be powered by AGNs. Furthermore, the majority of known optically selected AGNs in dwarf galaxies do not dominate the WISE colors.

Our results are consistent with evidence in the literature that the ionizing UV radiation from young stars is one of the primary sources of dust heating in low-metallicity dwarf galaxies (Izotov et al. 2014). Both IRAS and Spitzer observations demonstrated that dwarf galaxies have evidence for large quantities of hot dust (Helou 1986; Hunter et al. 1989; Melisse \& Israel 1994; Cannon et al. 2006; Rosenberg et al. 2006). In addition, it has been shown that the IR SED peak of dwarf galaxies is broader, which is often explained as resulting from dust at higher temperatures than what is observed in more massive galaxies (Boselli et al. 2012; Smith et al. 2012; RémyRuyer et al. 2013, 2015; Ciesla et al. 2014). The temperatures from the tracks in Figure 7 for the hot dust that would be necessary to produce the observed WISE colors has been observed in local low-metallicity galaxies from Spitzer $8 \mu \mathrm{m}$ observations (Engelbracht et al. 2005; Jackson et al. 2006), and even hotter dust has been invoked to explain the near-IR excesses observed in the dwarf galaxies SBS 0335-052 (Reines et al. 2008) and Haro 3 (Johnson et al. 2004). Recently, in an analysis of the infrared properties of a large sample of lowmetallicity dwarf galaxies, Rémy-Ruyer et al. (2015) demonstrated that the dust SED for these galaxies peaks at shorter wavelengths as compared to higher-metallicity systems, which they attribute to a clumpy interstellar medium that allows for a wider range of dust temperatures. In addition, the lower-metallicity dust will attenuate the light from young stars less, and the dust can then be heated deeper within individual molecular clouds. Cormier et al. (2015) used Herschel PACS spectroscopy of low-metallicity dwarf galaxies to provide evidence that the interstellar medium in these galaxies is more porous than in metal-rich galaxies, leading to a larger fraction of the stellar UV radiation heating dust. These results are supported by modeling by Hirashita \& Hunt (2004), who found that dust temperature and dust luminosity is higher in dense, compact, low-metallicity star-forming regions.

Support for A.E.R. was provided by NASA through Hubble Fellowship grant HST-HF2-51347.001-A awarded by the Space Telescope Science Institute, which is operated by the Association of Universities for Research in Astronomy, Inc., for NASA, under contract NAS 5-26555. The work of D.S. was carried out at the Jet Propulsion Laboratory, California Institute of Technology, under a contract with NASA. We are grateful to the entire SDSS collaboration for providing the data that made this work possible, and to Michael Blanton and all those involved in creating the NASA-Sloan Atlas. This publication makes use of data products from the Wide-field Infrared Survey Explorer, which is a joint project of the University of California, Los Angeles, and the Jet Propulsion Laboratory/ California Institute of Technology, and NEOWISE, which is a project of the Jet Propulsion Laboratory/California Institute of Technology. WISE and NEOWISE are funded by the National Aeronautics and Space Administration.

\section{APPENDIX \\ THUMBNAILS OF THE JARRETT ET AL. IR-AGN CANDIDATES WITHOUT OPTICAL EMISSION-LINE FLUX MEASUREMENTS}

The SDSS and WISE thumbnails for the 25 potential IRselected AGN dwarf galaxy candidates without optical emission-line flux measurements are shown in Figures 10-12.

\section{REFERENCES}

Aihara, H., Allende Prieto, C., An, D., et al. 2011, ApJS, 193, 29

Aird, J., Coil, A. L., Moustakas, J., et al. 2012, ApJ, 746, 90

Ashby, M. L. N., Stern, D., Brodwin, M., et al. 2009, ApJ, 701, 428

Assef, R. J., Kochanek, C. S., Brodwin, M., et al. 2010, ApJ, 713, 970

Baldassare, V. F., Reines, A. E., Gallo, E., et al. 2016, ApJ, 829, 57

Baldassare, V. F., Reines, A. E., Gallo, E., \& Greene, J. E. 2015, ApJL, 809, L14

Baldwin, J. A., Phillips, M. M., \& Terlevich, R. 1981, PASP, 93, 5

Barth, A. J., Ho, L. C., Rutledge, R. E., \& Sargent, W. L. W. 2004, ApJ, 607,90

Begelman, M. C., Volonteri, M., \& Rees, M. J. 2006, MNRAS, 370, 289

Bell, E. F., McIntosh, D. H., Katz, N., \& Weinberg, M. D. 2003, ApJS, 149,289

Bessiere, P. S., Tadhunter, C. N., Ramos Almeida, C., \& Villar Martín, M. 2012, MNRAS, 426, 276

Blanton, M. R., Kazin, E., Muna, D., Weaver, B. A., \& Price-Whelan, A. 2011, AJ, 142, 31

Blanton, M. R., \& Roweis, S. 2007, AJ, 133, 734

Bongiorno, A., Merloni, A., Brusa, M., et al. 2012, MNRAS, 427, 3103

Boselli, A., Ciesla, L., Cortese, L., et al. 2012, A\&A, 540, A54

Bromm, V., \& Yoshida, N. 2011, ARA\&A, 49, 373

Bruzual, G., \& Charlot, S. 2003, MNRAS, 344, 1000

Cannon, J. M., Walter, F., Armus, L., et al. 2006, ApJ, 652, 1170 
Ciesla, L., Boquien, M., Boselli, A., et al. 2014, A\&A, 565, A128

Cluver, M. E., Jarrett, T. H., Hopkins, A. M., et al. 2014, ApJ, 782, 90

Condon, J. J. 1992, ARA\&A, 30, 575

Cormier, D., Madden, S. C., Lebouteiller, V., et al. 2015, A\&A, 578, A53

Dong, X.-B., Ho, L. C., Yuan, W., et al. 2012, ApJ, 755, 167

Donley, J. L., Rieke, G. H., Pérez-González, P. G., \& Barro, G. 2008, ApJ, 687,111

Ellison, S. L., Patton, D. R., Mendel, J. T., \& Scudder, J. M. 2011, MNRAS, 418, 2043

Engelbracht, C. W., Gordon, K. D., Rieke, G. H., et al. 2005, ApJL, 628, L29 Engelbracht, C. W., Rieke, G. H., Gordon, K. D., et al. 2008, ApJ, 678, 804 Ferrarese, L., \& Merritt, D. 2000, ApJL, 539, L9

Filippenko, A. V., \& Ho, L. C. 2003, ApJL, 588, L13

Filippenko, A. V., \& Sargent, W. L. W. 1989, ApJL, 342, L11

Freitag, M., Gürkan, M. A., \& Rasio, F. A. 2006, MNRAS, 368, 141

Galliano, F., Dwek, E., \& Chanial, P. 2008, ApJ, 672, 214

Gebhardt, K., Bender, R., Bower, G., et al. 2000, ApJL, 539, L13

Giersz, M., Leigh, N., Hypki, A., Lützgendorf, N., \& Askar, A. 2015 , MNRAS, 454, 3150

Goswami, S., Umbreit, S., Bierbaum, M., \& Rasio, F. A. 2012, ApJ, 752, 43

Greene, J. E., \& Ho, L. C. 2004, ApJ, 610, 722

Greene, J. E., \& Ho, L. C. 2007, ApJ, 670, 92

Griffith, R. L., Tsai, C.-W., Stern, D., et al. 2011, ApJL, 736, L22

Gürkan, M. A., Freitag, M., \& Rasio, F. A. 2004, ApJ, 604, 632

Haehnelt, M. G., \& Rees, M. J. 1993, MNRAS, 263, 168

Hainline, K. N., Hickox, R. C., Carroll, C. M., et al. 2014, ApJ, 795, 124

Hainline, K. N., Shapley, A. E., Greene, J. E., et al. 2012, ApJ, 760, 74

Helou, G. 1986, ApJL, 311, L33

Hickox, R. C., Jones, C., Forman, W. R., et al. 2007, ApJ, 671, 1365

Hirashita, H., \& Hunt, L. K. 2004, A\&A, 421, 555

Hopkins, P. F., Hernquist, L., Cox, T. J., \& Kereš, D. 2008, ApJS, 175, 356

Hunter, D. A., Gallagher, J. S., III, Rice, W. L., \& Gillett, F. C. 1989, ApJ, 336,152

Izotov, Y. I., Guseva, N. G., Fricke, K. J., \& Henkel, C. 2011, A\&A, 536, L7

Izotov, Y. I., Guseva, N. G., Fricke, K. J., Krügel, E., \& Henkel, C. 2014, A\&A, 570, A97

Izotov, Y. I., Lipovetsky, V. A., Chaffee, F. H., et al. 1997, ApJ, 476, 698

Jackson, D. C., Cannon, J. M., Skillman, E. D., et al. 2006, ApJ, 646, 192

Jarrett, T. H., Cohen, M., Masci, F., et al. 2011, ApJ, 735, 112

Johnson, K. E., Indebetouw, R., Watson, C., \& Kobulnicky, H. A. 2004, AJ, 128,610

Kauffmann, G., Heckman, T. M., Tremonti, C., et al. 2003, MNRAS, 346, 1055

Kennicutt, R. C., \& Evans, N. J. 2012, ARA\&A, 50, 531

Kewley, L. J., Dopita, M. A., Leitherer, C., et al. 2013, ApJ, 774, 100

Kewley, L. J., Dopita, M. A., Sutherland, R. S., Heisler, C. A., \& Trevena, J. 2001, ApJ, 556, 121

Kormendy, J., \& Ho, L. C. 2013, ARA\&A, 51, 511

Kormendy, J., \& Richstone, D. 1995, ARA\&A, 33, 581

Koss, M., Mushotzky, R., Veilleux, S., \& Winter, L. 2010, ApJL, 716, L125

Lacy, M., Ridgway, S. E., Gates, E. L., et al. 2013, ApJS, 208, 24

Lacy, M., Storrie-Lombardi, L. J., Sajina, A., et al. 2004, ApJS, 154, 166

Leitherer, C., Schaerer, D., Goldader, J. D., et al. 1999, ApJS, 123, 3
Lemons, S. M., Reines, A. E., Plotkin, R. M., Gallo, E., \& Greene, J. E. 2015, ApJ, 805, 12

Lodato, G., \& Natarajan, P. 2006, MNRAS, 371, 1813

Lützgendorf, N., Kissler-Patig, M., Gebhardt, K., et al. 2016, in IAU Symp. 312, Star Clusters and Black Holes in Galaxies across Cosmic Time, ed. Y. Meiron et al. (Cambridge: Cambridge Univ. Press), 181

Madden, S. C., Galliano, F., Jones, A. P., \& Sauvage, M. 2006, A\&A, 446, 877

Mateos, S., Alonso-Herrero, A., Carrera, F. J., et al. 2012, MNRAS, 426, 3271

Melisse, J. P. M., \& Israel, F. P. 1994, A\&A, 285, 51

Mendez, A. J., Coil, A. L., Aird, J., et al. 2013, ApJ, 770, 40

Mezcua, M., Roberts, T. P., Lobanov, A. P., \& Sutton, A. D. 2015, MNRAS, 448, 1893

Moran, E. C., Shahinyan, K., Sugarman, H. R., Vélez, D. O., \& Eracleous, M. 2014, AJ, 148, 136

O’Connor, J. A., Rosenberg, J. L., Satyapal, S., \& Secrest, N. J. 2016, MNRAS, 463, 811

O’Donnell, J. E. 1994, ApJ, 422, 158

Pardo, K., Goulding, A. D., Greene, J. E., et al. 2016, ApJ, 831, 203

Polletta, M., Tajer, M., Maraschi, L., et al. 2007, ApJ, 663, 81

Reines, A., \& Comastri, A. 2016, arXiv:1609.03562

Reines, A. E., \& Deller, A. T. 2012, ApJL, 750, L24

Reines, A. E., Greene, J. E., \& Geha, M. 2013, ApJ, 775, 116

Reines, A. E., Johnson, K. E., \& Hunt, L. K. 2008, AJ, 136, 1415

Reines, A. E., Plotkin, R. M., Russell, T. D., et al. 2014, ApJL, 787, L30

Reines, A. E., Sivakoff, G. R., Johnson, K. E., \& Brogan, C. L. 2011, Natur, 470, 66

Reines, A. E., \& Volonteri, M. 2015, ApJ, 813, 82

Rémy-Ruyer, A., Madden, S. C., Galliano, F., et al. 2013, A\&A, 557, A95

Rémy-Ruyer, A., Madden, S. C., Galliano, F., et al. 2015, A\&A, 582, A121

Rosenberg, J. L., Ashby, M. L. N., Salzer, J. J., \& Huang, J.-S. 2006, ApJ, 636,742

Sabater, J., Best, P. N., \& Argudo-Fernández, M. 2013, MNRAS, 430, 638

Salpeter, E. E. 1955, ApJ, 121, 161

Sartori, L. F., Schawinski, K., Treister, E., et al. 2015, MNRAS, 454, 3722

Satyapal, S., Secrest, N. J., McAlpine, W., et al. 2014, ApJ, 784, 113

Schulze, A., \& Wisotzki, L. 2010, A\&A, 516, A87

Secrest, N. J., Satyapal, S., Gliozzi, M., et al. 2015, ApJ, 798, 38

Smith, D. J. B., Dunne, L., da Cunha, E., et al. 2012, MNRAS, 427, 703

Stasińska, G., \& Leitherer, C. 1996, ApJS, 107, 661

Stern, D., Assef, R. J., Benford, D. J., et al. 2012, ApJ, 753, 30

Stern, D., Eisenhardt, P., Gorjian, V., et al. 2005, ApJ, 631, 163

Trump, J. R., Sun, M., Zeimann, G. R., et al. 2015, ApJ, 811, 26

van der Marel, R. P., Alves, D. R., Hardy, E., \& Suntzeff, N. B. 2002, AJ, 124, 2639

van Wassenhove, S., Volonteri, M., Walker, M. G., \& Gair, J. R. 2010, MNRAS, 408, 1139

Wright, E. L., Eisenhardt, P. R. M., Mainzer, A. K., et al. 2010, AJ, 140, 1868

Wu, H., Cao, C., Hao, C.-N., et al. 2005, ApJL, 632, L79

Yan, R. 2011, AJ, 142, 153

Yan, R., \& Blanton, M. R. 2012, ApJ, 747, 61

York, D. G. \& SDSS Collaboration 2000, AJ, 120, 1579

Zibetti, S., Charlot, S., \& Rix, H.-W. 2009, MNRAS, 400, 1181 